OSTI mcs-P.-510-0495

\title{
Heterogeneous Domain Decomposition for Singularly Perturbed Elliptic Boundary Value Problems
}

\author{
Marc Garbey ${ }^{1}$ \\ LAN - URA 740, Université Claude Bernard Lyon 1 \\ 69622 Villeurbanne cedex, France \\ e-mail: mgarbey@lan1.univ-lyon1.fr \\ Hans G. Kaper ${ }^{2}$ \\ Mathematics and Computer Science Division \\ Argonne National Laboratory, Argonne, IL 60439, USA \\ e-mail: kaperomcs.anl.gov \\ April 14, 1995

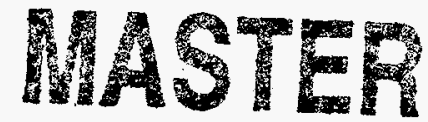

Proposed running head: Domain Decomposition for Singular Perturbation Problems

AMS (MOS) subject classification: Primary 35B25, 65N05; Secondary 35J25, 35J40

Key words: Elliptic boundary value problems, singular perturbations, boundary layers, transition layers, turning-point problems, asymptotic analysis, domain decomposition, iterative methods

DISTRIBUTION OF THIS DOCUMENT IS UNLIMITED

\footnotetext{
${ }^{1}$ Supported by the Fondation Cromey-le-Bas under contract BS-91-1bis.

${ }^{2}$ Supported by the Mathematical, Information, and Computational Sciences Division subprogram of the Office of Computational and Technology Research, U.S. Department of Energy, under Contract W-31-109Eng-38.
} 


\title{
Heterogeneous Domain Decomposition for Singularly Perturbed Elliptic Boundary Value Problems
}

\begin{abstract}
A heterogeneous domain-decomposition method is presented for the numerical solution of singularly perturbed elliptic boundary value problems. The method, which is parallelizable at various levels, uses several ideas of asymptotic analysis. The subdomains match the domains of validity of the local ("inner" and "outer") asymptotic expansions, and cut-off functions are used to match solutions in neighboring subdomains. The positions of the interfaces, as well as the mesh widths, depend on the small parameter, $\varepsilon$. On the subdomains, iterative solution techniques are used, which may vary from one subdomain to another. The global convergence rate depends on $\varepsilon$; it generally increases like some power of $\left(\log \left(\varepsilon^{-1}\right)\right)^{-1}$ as $\varepsilon \downarrow 0$. The method is illustrated on several two-dimensional singular perturbation problems.
\end{abstract}

\section{Introduction}

We are interested in the numerical solution of singularly perturbed second-order elliptic boundary value problems with Dirichlet boundary data, generically represented by the equations

$$
L(\varepsilon) u=f \text { on } \Omega, \quad u=g \text { on } \partial \Omega .
$$

Here, $\Omega$ is a bounded domain in $R^{d}$, whose boundary $\partial \Omega$ is Lipschitz continuous; $\varepsilon$ is a small positive parameter, $\varepsilon \in\left(0, \varepsilon_{0}\right)$ say, where $\varepsilon_{0}$ is arbitrarily small positive but fixed; $L(\varepsilon)$ is a differential expression, uniformly elliptic for every $\varepsilon \in\left(0, \varepsilon_{0}\right)$, which reduces formally to a lower-order differential expression $L(0)$ as $\varepsilon \downarrow 0$. The functions $f$ and $g$ are given; they are defined on $\Omega$ and $\partial \Omega$, respectively. We assume that (1.1) has a unique classical solution $u_{\Omega}(\varepsilon) \in C^{2}(\Omega) \cap C(\bar{\Omega})$ for every $\varepsilon \in\left(0, \varepsilon_{0}\right)$. The point values of $u_{\Omega}(\varepsilon)$ are $u_{\Omega}(\varepsilon)(x)=$ $u_{\Omega}(\varepsilon, x), x \in \bar{\Omega}$. The set of all solutions $u_{\Omega}(\varepsilon)$ defines a mapping $u_{\Omega}:\left(0, \varepsilon_{0}\right) \rightarrow X(\Omega)$.

Notation. In general, we use the symbol $X(\cdot)$ to denote a finite- or infinite-dimensional Banach space of functions defined on the argument set, endowed with the topology of uniform convergence.

Solutions of singular perturbation problems typically display rapid variations across narrow regions (boundary layers, transition layers, fronts). These variations pose obvious obstacles to an efficient numerical solution scheme. Although singular perturbation problems have been studied extensively in asymptotic analysis [1-7], the results of these studies have, at least so far, not found their way into computational algorithms. To the best of our knowledge, Chin et al. [8] were the first to suggest a domain-decomposition algorithm based on asymptotic arguments. Subsequent references addressing domain decomposition 
in the context of singular perturbation problems are [9-17]. Conference proceedings [18-23] are an excellent source for articles on domain decomposition in general, but they do not address the issues involved in the solution of singular perturbation problems.

In the present article, we construct, in a systematic way, a heterogeneous domaindecomposition method for the numerical solution of (linear) singularly perturbed elliptic boundary value problems. The method is inspired directly by the method of matched asymptotic expansions and incorporates several concepts of asymptotic analysis; for example, the subdomains correspond to the "regular domains" and the "singular layers," and solutions on neighboring subdomains are "matched" by means of cut-off functions. (We prefer, and will use, the terms "regular domain" and "singular layer," instead of the more arcane expressions "outer" and "inner" domain customary in asymptotic analysis.) The position of the interfaces depends on the small parameter, $\varepsilon$, and is determined in such a way that the truncation error is asymptotically of the same order on each subdomain. The domain decomposition is combined with a Schwarz alternating procedure, which alternates between the regular domains and the singular layers, and an iterative solution on each subdomain. The Schwarz procedure is implemented in its most elementary form: the subdomains overlap, and Dirichlet data are specified at the interfaces [24]. Thus, it is easy to couple different approximations on the various subdomains. The procedure requires the temporary specification of data in the interior of $\Omega$, and its convergence depends on the way these data propagate into the interior of the domain. As was shown in [25], the propagation factor can be made arbitrarily small for the most common finite-difference schemes, provided the mesh size is properly chosen as a function of $\varepsilon$. On each subdomain, the iterative method is designed to generate successive terms in the asymptotic expansion of the solution. By judiciously choosing the mesh widths as functions of the small parameter, we achieve convergence of the asymptotic expansions in the classical sense for fixed (but sufficiently small) values of $\varepsilon$. The iterative solver may vary from one subdomain to another, so the method is truly heterogeneous. The global convergence rate of the method generally improves like some power of $\left(\log \left(\varepsilon^{-1}\right)\right)^{-1}$ as $\varepsilon \downarrow 0$.

The algorithm was designed from the perspective of parallel computing. It offers parallelism at the large-granularity level (domain decomposition) and at the small-granularity level (subdomain solution). Moreover, it solves irregular structures such as boundary layers and transition layers on Cartesian grids with large aspect ratios. Thus, a regular data structure is maintained, and the potential for parallelism is preserved. Load balancing remains a critical issue in the parallel implementation. Even if the number of iterations per subdomain is chosen to match the estimated convergence rate, different numbers of processors may have to be assigned to different subdomains to balance the cost of the iterative solvers. Some of the ideas described in this article have been implemented and used to solve nonlinear boundary value problems on MIMD architectures [26-28].

The method is described in detail in Section 2 and illustated on a singularly perturbed diffusion problem (Section 3), a singularly perturbed convection problem (Section 4), and 
a turning-point problem (Section 5). Several relevant concepts of asymptotic analysis are summarized in Appendix A.

\section{Description of the Method}

In singular peturbation problems of the type (1.1), the domain $\Omega$ decomposes naturally into regular subdomains and singular layers. A singular layer may be situated in the interior of $\Omega$ between two regular subdomains (transition layer) or adjacent to (a part of) the boundary $\partial \Omega$ (boundary layer).

By definition, a regular subdomain is a subset of $\Omega$, where the solution admits a regular asymptotic expansion. The expansion is an asymptotic approximation to the solution on the regular subdomain. Characteristically, the expansion does not extend to an asymptotic approximation on the entire domain, because of the presence of singular layers. However, in a singular layer there usually exist special local variables (layer variables), which are scaled versions of the original variables, in terms of which the solution does have a regular asymptotic expansion. The scaling factor defining the layer variables depends on the small parameter. It then becomes a matter of matching this local expansion to the regular asymptotic expansion in the neighboring regular domain. The idea of decomposing the domain into regular subdomains and singular layers, constructing asymptotic expansions on each subdomain, and matching expansions across interfaces is at the heart of the method presented in this article.

Before describing the method proper, we illustrate the ideas on a simple example,

$$
L(\varepsilon) u \equiv-\varepsilon \Delta u+u=f \text { on } \Omega, u=g \text { on } \partial \Omega,
$$

where $g$ is the trace of $f$ on $\partial \Omega$. In this case, there are no singular layers, and the solution $u_{\Omega}(\varepsilon)$ admits an asymptotic approximation on the entire domain $\Omega$. The approximation has the form of a regular asymptotic expansion in powers of $\varepsilon$. That is, there exist functions $v_{i}$ that do not depend on $\varepsilon$, such that the difference between $u_{\Omega}(\varepsilon)$ and the partial sum $u^{(n)}(\varepsilon)=\sum_{i=0}^{n} \varepsilon^{i} v_{i}$ vanishes as $\varepsilon \downarrow 0$ for each (fixed) $n, n=0,1, \ldots$ The functions $v_{i}$ are obtained recursively; $v_{0}=f$ on $\Omega$, and $v_{1}, v_{2}, \ldots$ are found by solving the boundary value problem $v_{i}=\Delta v_{i-1}$ on $\Omega, v_{i}=0$ on $\partial \Omega$, successively for $i=1,2, \ldots$. Thus, the regular asymptotic expansion is generated by the iterative procedure

$$
u^{(n)}=f+\varepsilon \Delta u^{(n-1)} \text { on } \Omega, u^{(n)}=0 \text { on } \partial \Omega ; \quad n=1,2, \ldots,
$$

starting with $u^{(0)}=f$. The expansion does not converge in the classical sense, as $n \rightarrow \infty$, because the Laplacian defines an unbounded operator in the underlying vector space.

The concept of asymptotic convergence, on which the preceding argument is based, is not very useful for computational purposes. Because $\varepsilon$ is given, it cannot be made arbitrarily 
small, and if $\varepsilon$ is fixed, we do not have convergence as $n \rightarrow \infty$. But we claim that the discrete analog of (2.2) is worth considering. Suppose $\Omega_{h}$ is the computational grid on $\Omega$ and $\Delta_{h}$ is the discrete approximation to the Laplacian on $\Omega_{h}$. The discrete analog of (2.2) is

$$
u^{(n)}=f_{h}+\varepsilon \Delta_{h} u^{(n-1)} \text { on } \Omega_{h}, u^{(n)}=0 \text { on } \partial \Omega_{h} ; \quad n=1,2, \ldots,
$$

with $u^{(0)}=f_{h}$. It generates a sequence, $\left\{u^{(n)}(\varepsilon, h): n=0,1, \ldots\right\}$, of vectors $u^{(n)}(\varepsilon, h)$ on $\Omega_{h}$. Whether this sequence converges as $n \rightarrow \infty$ depends on the norm of the operator defined by the expression $\varepsilon \Delta_{h}$ on the underlying vector space. For most approximation schemes, the discrete Laplacian $\Delta_{h}$ defines an operator whose norm is bounded by some (negative) power of the mesh width, $h^{-p}$ say. Suppose that $h$ is allowed to vary with $\varepsilon$, and suppose, in particular, that $\varepsilon(h(\varepsilon))^{-p}=o(1)$ as $\varepsilon \downarrow 0$. The mapping defined by $\varepsilon \Delta_{h}$ is then a contraction for all sufficiently small $\varepsilon$, and the sequence $\left\{u^{(n)}(\varepsilon, h(\varepsilon)): n=0,1, \ldots\right\}$ converges in the classical sense (that is, as $n \rightarrow \infty$ ) for each sufficiently small, but fixed, $\varepsilon$. The limiting function, $u(\varepsilon, h(\varepsilon))$, is the solution of the discrete analog of $(2.1)$ on the grid $\Omega_{h(\varepsilon)}$,

$$
L(\varepsilon, h(\varepsilon)) u \equiv-\varepsilon \Delta_{h(\varepsilon)} u+u=f_{h(\varepsilon)} \text { on } \Omega_{h(\varepsilon)}, u=g_{h(\varepsilon)} \text { on } \partial \Omega_{h(\varepsilon)} .
$$

The truncation error-the distance between the trace of $u_{\Omega}(\varepsilon)$ on $\Omega_{h(\varepsilon)}$ and $u(\varepsilon, h(\varepsilon))$-can be estimated in terms of the mesh width $h(\varepsilon)$ by the usual methods of numerical analysis. The discrete iterative procedure (2.3) thus leads to a computationally useful algorithm.

The example shows that the crucial ingredient of the method is the boundedness of $\Delta_{h}$. Whereas the Laplacian $\Delta$ leads to an unbounded operator, its discrete approximation $\Delta_{h}$ defines a bounded operator. The bound may (and does) depend on the mesh width $h$, but if $h$ depends on the small parameter, $\varepsilon$, and we choose the functional dependence asymptoptically speaking "just right" as $\varepsilon \downarrow 0$, we generate a sequence of approximations that converges in the classical sense for each fixed (but sufficiently small) $\varepsilon$. In essence, we propose to take limits along certain admissible curves $S=\left\{(\varepsilon, h(\varepsilon)): \varepsilon \in\left(0, \varepsilon_{0}\right)\right\}$ in the $(\varepsilon, h)$-plane. All admissible curves terminate in the origin within an asymptotically prescribed cone as $\varepsilon \downarrow 0$. Thus, while asymptotic convergence is concerned with limits along lines of constant $h$ and classical convergence with limits along lines of constant $\varepsilon$, we propose a new paradigm by combining aspects of both. Given this idea, the remaining difficulties are primarily technical: How to design efficient iterative solution techniques on the subdomains, and how to combine the expansions generated by the iterative solvers on the subdomains into a global solution on the entire domain.

\subsection{Domain Decomposition}

Consider the singular perturbation problem (1.1). To miminize the complexity of the presentation, we assume that the problem has a single boundary layer, which is adjacent to a smooth part $\Gamma$ (the singular part) of $\partial \Omega$. More complicated situations can be handled by a divide-and-conquer technique, as will be evident from the example in Section 5. Generally, 
$\Gamma$ is a manifold of codimension $d^{\prime}$, where $d^{\prime}$ may be any integer less than or equal to $d$. If $d=2$, the two possible configurations correspond to a (true) boundary layer $\left(d^{\prime}=1\right)$ and a corner layer $\left(d^{\prime}=2\right)$.

As a first step, we decompose $\Omega$ into two overlapping subdomains,

$$
\Omega=\Omega_{0} \cup \Omega_{1}, \Omega_{0} \cap \Omega_{1} \neq \emptyset \text {. }
$$

The index $i$ identifies the subdomain; our convention is that $i=0$ refers to the regular subdomain, $i=1,2, \ldots$ to the singular layers. (In this case, there is only one singular layer, so $i=1$.) The boundaries $\partial \Omega_{0}$ and $\partial \Omega_{1}$ coincide partially with $\partial \Omega$; the complementary parts are the interfaces $\Gamma_{0}$ and $\Gamma_{1}$,

$$
\partial \Omega_{i}=\left(\partial \Omega_{i} \cap \partial \Omega\right) \cup \Gamma_{i}, \quad \Gamma_{i}=\partial \Omega_{i} \backslash \overline{\left(\partial \Omega_{i} \cap \partial \Omega\right)}, \quad i=0,1 .
$$

Thus, $\Gamma_{0} \subset \Omega_{1}$ and $\Gamma_{1} \subset \Omega_{0}$. The position of the interfaces may vary with $\varepsilon$. Note that the singular part of the boundary, $\Gamma$, is a subset of $\partial \Omega_{1} \backslash \Gamma_{1}$.

The decomposition (2.5) must satisfy the following asymptotic relations as $\varepsilon \downarrow 0$ :

$$
\begin{gathered}
\operatorname{diam}_{j}\left(\Omega_{0}\right)=O^{\sharp}(1), j=1, \ldots, d ; \\
\operatorname{diam}_{j}\left(\Omega_{1}\right)=o(1), j=1, \ldots, d^{\prime} ; \operatorname{diam}_{j}\left(\Omega_{1}\right)=O^{\sharp}(1), j=d^{\prime}+1, \ldots, d ; \\
\operatorname{diam}_{j}\left(\Omega_{1}\right) \approx \operatorname{dist}_{j}\left(\Gamma, \Gamma_{0}\right), j=1, \ldots, d^{\prime} .
\end{gathered}
$$

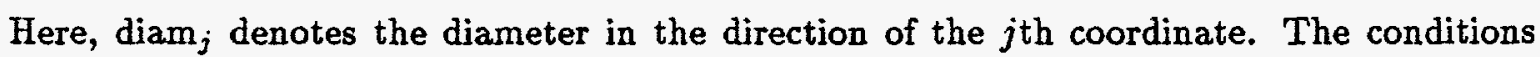
(2.7) and (2.8) reflect the fact that the boundary layer becomes infinitesimally thin in the first $d^{\prime}$ coordinate directions as $\varepsilon \downarrow 0$. The condition (2.9) implies that, in each direction transverse to $\Gamma$, the diameter of $\Omega_{1}$ remains comparable to the distance from $\Gamma$ to $\Gamma_{0}$ as $\varepsilon \downarrow 0$.

The decomposition (2.5) induces a decomposition of the boundary value problem (1.1),

$$
L(\varepsilon) u=f_{i} \text { on } \Omega_{i}, u=g_{i} \text { on } \partial \Omega_{i}, \quad i=0,1,
$$

where

$$
f_{i}=\left.f\right|_{\Omega_{i}}, g_{i}= \begin{cases}g & \text { on } \partial \Omega_{i} \backslash \Gamma_{i}, \\ \gamma_{i} u_{\Omega}(\varepsilon) & \text { on } \Gamma_{i} .\end{cases}
$$

Here, $\gamma_{i}$ is the trace operator on $\Gamma_{i}$. The solution of $(2.10)$, which we denote by $u_{i}(\varepsilon)$, coincides with the restriction of $u_{\Omega}(\varepsilon)$ to $\Omega_{i}$,

$$
u_{i}(\varepsilon)=\left.u_{\Omega}(\varepsilon)\right|_{\Omega_{i}}
$$




\subsection{Discretization Procedure}

For the numerical solution of (1.1), we impose a uniform grid on $\bar{\Omega}$. To avoid the need for interpolation on the interfaces, we assume that this grid conforms with the overlap region $\Omega_{0} \cap \Omega_{1}$. We refine this grid uniformly in the boundary layer. Let $\Omega_{h 0}$ denote the set of all points of the coarse grid that belong to $\Omega_{0}$ and its boundary $\partial \Omega_{0}$, and $\Omega_{h 1}$ the set of all points of the fine grid that belong to $\Omega_{1}$ and its boundary $\partial \Omega_{1}$. If necessary, we distinguish between points of $\Omega_{h i}(i=0,1)$ that lie inside $\Omega_{i}$ (also denoted by $\Omega_{h i}$ ) and points that lie on the boundary $\partial \Omega_{i}$ of $\Omega_{i}$ (denoted by $\partial \Omega_{h i}$ ). The composite grid is $\Omega_{h}=\Omega_{h 0} \cup \Omega_{h 1}$.

On each grid $\Omega_{h i}$, the mesh widths generally vary from one coordinate direction to another; hence, $\Omega_{h i}$ is characterized by a vector of mesh widths $h_{i}=\left(h_{i, 1}, \ldots, h_{i, d}\right)$. The mesh widths may depend on $\varepsilon$, but we impose the restriction that, in any direction (index $j$ ), the number of grid points on each subdomain (index $i$ ) is asymptotically of the same order as $\varepsilon \downarrow 0$,

$$
\frac{\operatorname{diam}_{j}\left(\Omega_{i}\right)}{h_{i, j}} \approx N_{j}, \quad j=1, \ldots, d .
$$

The quantities $N_{j}$ may depend on $\varepsilon$, but not on $i$.

We approximate each boundary value problem (2.10) by a discrete problem,

$$
L_{i}\left(\varepsilon, h_{i}\right) u=f_{i} \text { on } \Omega_{h i}, u=g_{i} \text { on } \partial \Omega_{h i}, \quad i=0,1 \text {. }
$$

The order of the approximation may vary by subdomain, and even by direction within a subdomain, so the procedure is truly heterogeneous. We denote the solution of (2.14) by $u_{i}\left(\varepsilon, h_{i}\right)$.

The accuracy of the discretization procedure is measured by the truncation error. We assume that there exist (positive) integers $\left(k_{j}, l_{j}\right)$ such that, on each subdomain $\Omega_{i}$,

$$
\left\|\left.u_{i}(\varepsilon)\right|_{\Omega_{h i}}-u_{i}\left(\varepsilon, h_{i}\right)\right\|_{X\left(\Omega_{h s}\right)}=O\left(\sum_{j=1}^{d} h_{i, j}^{k_{j}}\left(\operatorname{diam}_{j}\left(\Omega_{i}\right)\right)^{l_{j}}\left\|\partial_{j}^{k_{j}+l_{j}} u_{i}\right\|_{X\left(\Omega_{i}\right)}\right),
$$

as $\varepsilon \downarrow 0$. The exact values of $k_{j}$ and $l_{j}$ are unimportant; eventually, these integers are absorbed in the constants in the asymptotic equivalence relation of Lemma 1 below. Hence, if they vary from one subdomain to the next, it suffices to identify $k_{j}$ and $l_{j}$ with the largest among the integers $k_{i, j}$ and $l_{i, j}$, respectively. We also subsume all mixed partial derivatives under the highest-order partial derivative in each direction.

\subsection{Truncation Error}

The domain-decomposition and discretization procedure leads to an asymptotically balanced approximation scheme if the condition (2.13) is satisfied and if, in addition, the truncation 
errors on the subdomains are asymptotically of the same order as $\varepsilon \downarrow 0$. The latter condition imposes a constraint on the choice of the interfaces.

Lemma 1 Suppose the transformation that regularizes (1.1) in the boundary layer stretches the $j$ th coordinate by a factor $\varepsilon^{-\nu_{j}}$. Then the domain decomposition (2.5), combined with the discretization procedure (2.14), results in an asymptotically balanced approximation scheme if $\operatorname{diam}_{j}\left(\Omega_{1}\right) \approx \varepsilon^{\nu_{j}} \log \left(\varepsilon^{-1}\right)$.

Proof. The proof requires an evaluation of the estimates $(2.15)$. We introduce the notation

$$
a_{j}=\operatorname{diam}_{j}\left(\Omega_{1}\right), b_{j}=\operatorname{dist}_{j}\left(\Gamma, \Gamma_{0}\right),
$$

for $j=1, \ldots, d^{\prime}$. Because $\Omega_{0} \cap \Omega_{1}$ has a nonempty interior, we have $b_{j}<a_{j}$. The numbers $a_{j}$ and $b_{j}$ depend on $\varepsilon$; the conditions (2.7) and (2.8) imply that $a_{j}=o(1), b_{j}=o(1)$, and $a_{j} \approx b_{j}$ for $j=1, \ldots, d^{\prime}$ as $\varepsilon \downarrow 0$. If convenient, we extend the definition of $a_{j}$ by taking $a_{j}=1$ for $j=d^{\prime}+1, \ldots, d$.

With $h_{j}=1 / N_{j}$, it follows from (2.13) that $h_{0, j} \approx h_{j}$ and $h_{1, j} \approx a_{j} h_{j}$.

On $\Omega_{0}$, the regular domain, the solution $u_{0}(\varepsilon)$ is the sum of an $n$-term asymptotic expansion and a boundary layer correction. The $n$-term asymptotic expansion defines a smooth function of position, which is $O^{\sharp}(1)$ and whose derivatives are $O(1)$ as $\varepsilon \downarrow 0$. On the scale of the boundary layer variables $(\xi)$, the boundary layer correction and its derivatives decay exponentially with the distance from $\Gamma$. Since $\Gamma$ is at least a distance $b_{j}$ away from any point of $\Omega_{0}$, we have $\xi_{j} \geq b_{j} \varepsilon^{-\nu_{j}}$. Any derivative with respect to $x_{j}$ corresponds to $\varepsilon^{-\nu_{j}}$ times the derivative with respect to $\xi_{j}$. Hence, $\partial_{j}^{m} u_{0}(\varepsilon)=O\left(1+\varepsilon^{-\nu_{j} m} \exp \left(-\gamma_{j} \varepsilon^{-\nu_{j}} b_{j}(\varepsilon)\right)\right)$ for some positive constant $\gamma_{j}$, and

$$
\left\|\partial_{j}^{k_{j}+l_{j}} u_{0}\right\|_{X\left(\Omega_{0}\right)}=O\left(1+\varepsilon^{-\nu_{j}\left(k_{j}+l_{j}\right)} \exp \left(-\gamma_{j} \varepsilon^{-\nu_{j}} b_{j}(\varepsilon)\right)\right) .
$$

Next, consider the solution $u_{1}(\varepsilon)$ on $\Omega_{1}$. The transformation from the original variables $(x)$ to the boundary layer variables $(\xi)$ sends $u_{1}(\varepsilon)$ into a function $U_{1}(\varepsilon)$, which is $O^{\sharp}(1)$ and whose partial derivatives (with respect to the variables $\xi_{j}$ ) are $O(1)$ as $\varepsilon \downarrow 0$. Every derivative with respect to $x_{j}$ corresponds to $\varepsilon^{-\nu_{j}}$ times a derivative with respect to $\xi_{j}$. Hence, $\partial_{j}^{m} u_{1}=O\left(\varepsilon^{-\nu_{j} m}\right)$, and

$$
\left\|\partial_{j}^{k_{j}+l_{j}} u_{1}\right\|_{X\left(\Omega_{1}\right)}=O\left(\varepsilon^{-\nu_{j}\left(k_{j}+l_{j}\right)}\right) .
$$

Using the results (2.17) and (2.18) in (2.15), we find

$$
\left\|\left.u_{0}(\varepsilon)\right|_{\Omega_{h 0}}-u_{0}\left(\varepsilon, h_{0}\right)\right\|_{X\left(\Omega_{h 0}\right)}=O\left(\sum_{j=1}^{d} h_{j}^{k_{j}}\left(1+\varepsilon^{-\nu_{j}\left(k_{j}+l_{j}\right)} \exp \left(-\gamma_{j} \varepsilon^{-\nu_{j}} b_{j}(\varepsilon)\right)\right)\right)
$$




$$
\left\|\left.u_{1}(\varepsilon)\right|_{\Omega_{h 1}}-u_{1}\left(\varepsilon, h_{1}\right)\right\|_{X\left(\Omega_{h 1}\right)}=O\left(\sum_{j=1}^{d} h_{j}^{k_{j}}\left(\varepsilon^{-\nu_{j}} a_{j}(\varepsilon)\right)^{k_{j}+l_{j}}\right) .
$$

These estimates show that the order of the truncation error decreases exponentially with the order of $b_{j}$ on $\Omega_{0}$ and increases algebraically with the order of $a_{j}$ on $\Omega_{1}$. If $a_{j} \approx b_{j}$, as required, then the truncation errors balance if

$$
\left(\varepsilon^{-\nu_{j}} a_{j}(\varepsilon)\right)^{k_{j}+l_{j}} \approx 1+\varepsilon^{-\nu_{j}\left(k_{j}+l_{j}\right)} \exp \left(-\gamma_{j} \varepsilon^{-\nu_{j}} a_{j}(\varepsilon)\right) .
$$

This asymptotic equivalence relation holds if $\varepsilon^{-\nu j} a_{j}(\varepsilon) \approx \log \left(\varepsilon^{-1}\right)$.

We summarize the results of this and the preceding section in the following theorem.

Theorem 1 (Accuracy) Suppose that the domain decomposition (2.5) satisfies the conditions (2.7), (2.8), and (2.9) and that the grids imposed on the subdomains satisfy the constraint (2.13). If the approximation scheme is asymptotically balanced, then

$$
\left\|\left.u_{\Omega}(\varepsilon)\right|_{\Omega_{h}}-u(\varepsilon, h)\right\|_{X\left(\Omega_{h}\right)}=O\left(\sum_{j=1}^{d} N_{j}^{-k_{j}} \log \left(\varepsilon^{-1}\right)\right) .
$$

Note that we have lumped all powers of the logarithm into one. The estimate (2.22) is meant to convey the fact that the truncation error is determined primarily by a characteristic mesh width, $h_{j}=N_{j}^{-1}$, which generally vanishes like a power of $\varepsilon$, but the accuracy is mitigated somewhat by a logarithmic correction factor.

\subsection{Schwarz Alternating Procedure}

The decomposition (2.10) leads in a natural way to a simple Schwarz alternating procedure, where one solves a Dirichlet boundary value problem alternatingly on $\Omega_{0}$ and $\Omega_{1}$. The procedure, though simple, is particularly suited to heterogeneous methods, where different solution techniques, or even different representations of the boundary value problems, may be used on different subdomains. Moreover, the simple Schwarz alternating procedure, when implemented with overlapping subdomains, constitutes a direct analog of the matching procedure of asymptotic analysis.

The discussion in this section applies equally well to the solution of the continuous boundary value problem (2.10) and to the solution of its discrete analog, (2.14). We adopt the continuous point of view, even though we will apply the results later to the discrete case. Since the parameter $\varepsilon$ does not play a role in the present section, we suppress it temporarily. 
The Schwarz alternating procedure for $(2.10)$ is

$$
L_{0} u=f_{0} \text { on } \Omega_{0}, u=g_{0}^{(m)} \text { on } \partial \Omega_{0} ; \quad L_{1} u=f_{1} \text { on } \Omega_{1}, u=g_{1}^{(m)} \text { on } \partial \Omega_{1} ; \quad m=0,1, \ldots
$$

The inhomogeneous terms $f_{0}$ and $f_{1}$ do not vary throughout the iterative process,

$$
f_{i}=\left.f\right|_{\Omega_{i}}, \quad i=0,1 .
$$

The boundary data $g_{0}^{(m)}$ and $g_{1}^{(m)}$, on the other hand, vary from one step to the next, as they depend on the trace of the solution of the preceding boundary value problem on the interface. Let $\gamma_{i}$ denote the trace on $\Gamma_{i}(i=0,1)$.

At the initial step $(m=0)$, we take $g_{0}^{(0)}=g$ on $\partial \Omega_{0} \backslash \Gamma_{0}$ and assign arbitrary values to $g_{0}^{(0)}$ on $\Gamma_{0}$. Having found the solution, $u_{0}^{(0)}$, of the boundary value problem (2.23) on $\Omega_{0}$, we take $g_{1}^{(0)}=g$ on $\partial \Omega_{1} \backslash \Gamma_{1}$ and $g_{1}^{(0)}=\gamma_{1} u_{0}^{(0)}$ on $\Gamma_{1}$ and determine the solution, $u_{1}^{(0)}$, of the boundary value problem (2.23) on $\Omega_{1}$. After this initial step, we proceed successively for $m=1,2, \ldots$, taking

$$
g_{0}^{(m)}=\left\{\begin{array}{ll}
g & \text { on } \partial \Omega_{0} \backslash \Gamma_{0}, \\
\gamma_{0} u_{1}^{(m-1)} & \text { on } \Gamma_{0},
\end{array} g_{1}^{(m)}= \begin{cases}g & \text { on } \partial \Omega_{1} \backslash \Gamma_{1}, \\
\gamma_{1} u_{0}^{(m)} & \text { on } \Gamma_{1} .\end{cases}\right.
$$

The convergence of the Schwarz method depends essentially on the way errors introduced on the interfaces propagate into the subdomains.

Consider the boundary value problem

$$
L_{0} e=0 \text { on } \Omega_{0}, e= \begin{cases}0 & \text { on } \partial \Omega_{0} \backslash \Gamma_{0}, \\ \gamma_{0} e_{1} & \text { on } \Gamma_{0},\end{cases}
$$

where $e_{1} \in X\left(\Omega_{1}\right)$ is given. The solution of this boundary value problem is an element $e_{0} \epsilon$ $X\left(\Omega_{0}\right)$, whose trace on $\Gamma_{1}$ is $\gamma_{1} e_{0}$. Thus, (2.26) defines a mapping $Z_{0} \in \mathcal{L}\left(X\left(\Gamma_{0}\right), X\left(\Gamma_{1}\right)\right)$,

$$
Z_{0}: \gamma_{0} e_{1} \mapsto \gamma_{1} e_{0}, e_{1} \in X\left(\Omega_{1}\right) \text {. }
$$

The operator norm of this mapping defines a propagation number,

$$
\zeta_{0}=\left\|Z_{0}\right\|_{\mathcal{L}\left(X\left(\Gamma_{0}\right), X\left(\Gamma_{1}\right)\right)}=\sup \left\{\frac{\left\|\gamma_{1} e_{0}\right\|_{X\left(\Gamma_{1}\right)}}{\left\|\gamma_{0} e_{1}\right\|_{X\left(\Gamma_{0}\right)}}: e_{1} \in X\left(\Omega_{1}\right)\right\} .
$$

In the same way, we obtain a mapping $Z_{1} \in \mathcal{L}\left(X\left(\Gamma_{0}\right), X\left(\Gamma_{1}\right)\right)$ by considering the boundary value problem

$$
L_{1} e=0 \text { on } \Omega_{1}, e= \begin{cases}0 & \text { on } \partial \Omega_{1} \backslash \Gamma_{1}, \\ \gamma_{1} e_{0} & \text { on } \Gamma_{1},\end{cases}
$$

where $e_{0} \in X\left(\Omega_{0}\right)$ is given. If $e_{1} \in X\left(\Omega_{1}\right)$ is the solution of (2.29), then

$$
Z_{1}: \gamma_{1} e_{0} \mapsto \gamma_{0} e_{1}, e_{0} \in X\left(\Omega_{0}\right) \text {. }
$$


The corresponding propagation number is

$$
\zeta_{1}=\left\|Z_{1}\right\|_{\mathcal{L}\left(X\left(\Gamma_{1}\right), X\left(\Gamma_{0}\right)\right)}=\sup \left\{\frac{\left\|\gamma_{0} e_{1}\right\|_{X\left(\Gamma_{0}\right)}}{\left\|\gamma_{1} e_{0}\right\|_{X\left(\Gamma_{1}\right)}}: e_{0} \in X\left(\Omega_{0}\right)\right\} .
$$

The product of the two propagation numbers (2.28) and (2.31) defines the propagation factor of the Schwarz alternating procedure,

$$
\zeta=\zeta_{0} \zeta_{1} \text {. }
$$

The following lemma establishes the condition for convergence of the Schwarz alternating procedure.

Lemma 2 If $L_{0}$ and $L_{1}$ satisfy a maximum principle, and the propagation factor of the Schwarz alternating procedure satisfies the inequality $\zeta<1$, then

$$
\lim _{m \rightarrow \infty}\left\|u_{i}-u_{i}^{(m)}\right\|_{X\left(\Omega_{i}\right)}=0, i=0,1
$$

Proof. Let $e_{i}^{(m)}=u_{i}-u_{i}^{(m)}$ denote the error on $\Omega_{i}$ at the $m$ th step of the Schwarz alternating procedure. Because of the linearity, $e_{0}^{(m)}$ satisfies $(2.26)$ with $e_{1}=e_{1}^{(m-1)}$, and $e_{1}^{(m)}$ satisfies (2.29) with $e_{0}=e_{0}^{(m)}$. Hence, $\gamma_{1} e_{0}^{(m)} \leq \zeta_{0} \zeta_{1} \gamma_{1} e_{0}^{(m-1)}$ and $\gamma_{0} e_{1}^{(m)} \leq$ $\zeta_{0} \zeta_{1} \gamma_{0} e_{1}^{(m-1)}$, so the errors on $\Gamma_{0}$ and $\Gamma_{1}$ are reduced by a factor $\zeta$. If $\zeta<1$, convergence on $\Gamma_{0}$ and $\Gamma_{1}$ follows. If, in addition, $L$ satisfies the maximum principle, the convergence extends to $\Omega_{0}$ and $\Omega_{1}$.

Recall that we have suppressed the parameter $\varepsilon$ throughout the preceding discussion. The propagation numbers and the propagation factor do indeed depend on $\varepsilon$. In the discrete case, the propagation factor depends on the mesh widths $h_{i}$ as well.

\subsection{Iterative Solution on Subdomains}

We now turn to the solution of the boundary value problems (2.10) and their discrete analogs (2.14). We propose to use domain-dependent iterative solvers that generate successive terms of the asymptotic expansions on the respective subdomains. The iterative procedures are implemented within each step of the Schwarz alternating procedure; the index $m$ ( $m=$ $1,2, \ldots)$ refers to the current Schwarz step.

The following discussion applies again equally well to the continuous and the discrete case. In the continuous case, the fundamental concepts are not obscured by technical details of notation, so we adopt this point of view; eventually, however, we will apply the results to the discrete case only. 
In general, the iterative method on $\Omega_{;}$is based on a splitting of the differential expression

$$
L(\varepsilon)=L_{i, 0}(\varepsilon)+L_{i, 1}(\varepsilon) \text { on } \Omega_{i}, i=0,1 .
$$

The iterative method is

$$
L_{i, 0}(\varepsilon) u^{(m, n)}=f_{i}-L_{i, 1}(\varepsilon) u^{(m, n-1)} \text { on } \Omega_{i}, u^{(m, n)}=g_{i}^{(m)} \text { on } \partial \Omega_{i}, \quad n=1,2, \ldots,
$$

so $L_{i, 0}(\varepsilon)$ represents the implicit part, $L_{i, 1}(\varepsilon)$ the explicit part of $L(\varepsilon)$. We denote the solution of $(2.35)$ by $u_{i}^{(m, n)}(\varepsilon)$.

The choice of the components $L_{i, 0}$ and $L_{i, 1}$ is critical. As a general rule, $L_{i, 0}$ contains the terms that generate the dominant part of the asymptotic expansion of $u_{i}$. On the regular domain, these terms are found directly, in the boundary layer, after a transformation to the boundary layer variables. For example, in the problem (2.1) and the iterative procedure (2.2), we took $L_{i, 0}(\varepsilon)$ equal to the identity and $L_{i, 1}(\varepsilon)=-\varepsilon \Delta$. (Recall that we are really interested only in the discrete case, so read $\Delta_{h}$ instead of $\Delta$.) As a result, the iterative procedure (2.2) generated successive partial sums of the regular asymptotic expansion. The best we can say at this point is that the expansion converges asymptotically to $u_{i}$ as $\varepsilon \downarrow 0$. However, we will argue that the expansion actually converges in the classical sense for each fixed (but sufficiently small) $\varepsilon$. We will also see that the iterative procedure does not suffer from the usual instability associated with any procedure that treats higher-order derivatives explicitly.

The loss of boundary conditions introduces a complication on the regular domain, $\Omega_{0}$. In principle, we identify $L_{0,0}(\varepsilon)$ with the formal limit $L(0)$ of $L(\varepsilon)$ as $\varepsilon \downarrow 0$. But as $L(0)$ is of lower order than $L(\varepsilon)$, the boundary data on $\partial \Omega_{0}$ are incompatible with $L_{0,0}(\varepsilon)$, and a correction is needed. We accomplish this correction by means of a cut-off function, $\chi:\left(0, \varepsilon_{0}\right) \rightarrow C^{\infty}(\Omega)$, which coincides with the unit function on the overlap region, $\Omega_{0} \cap \Omega_{1}$, and whose support is asymptotically equivalent with $\overline{\Omega_{0} \cap \Omega_{1}}$ as $\varepsilon \downarrow 0$. The cut-off function serves two purposes. First, it enables us to formulate a well-posed boundary value problem on the regular domain. Second, when implemented in combination with a discretization of the differential expressions $L_{i, 0}(\varepsilon)$ and $L_{i, 1}(\varepsilon)$, it accomplishes the numerical analog of asymptotic matching for the Schwarz alternating procedure.

After these general remarks, we turn to the specifics. On the regular domain, $\Omega_{0}$, we define the components in (2.34) formally by

$$
L_{0,0}(\varepsilon)=L(0)+\chi(\varepsilon)(L(\varepsilon)-L(0)), \quad L_{0,1}(\varepsilon)=(1-\chi(\varepsilon))(L(\varepsilon)-L(0)),
$$

where $\chi$ is a cut-off function of the type discussed earlier. Thus, in (and possibly a little beyond) the overlap region $\Omega_{0} \cap \Omega_{1}, L_{0,0}$ is the same as $L(\varepsilon)$, but everywhere else in $\Omega_{0}$ it is the formal limit $L(0)$. The decomposition (2.36) is somewhat ad hoc, in the sense that the resulting solution need not be twice continuously differentiable everywhere in $\Omega_{0}$, but the method appears to work in practical problems, provided one has some knowledge 
of the position of the boundary and transition layers. If the position of the layers is not known, one can use a variant of the $\chi$ method developed for the Navier-Stokes equations [10, 29]. Note, however, that in the present method the higher-order derivatives are preserved, although the treatment depends on their order of magnitude, whereas normally in the $\chi$ method higher-order derivatives are dropped. Consequently, one is not faced with the usual difficulty of justifying the $\chi$ method, nor with the fact that it is difficult to say whether the method will work or not.

In the boundary layer, $\Omega_{1}$, we define the components in (2.34) indirectly by considering the boundary value problem in terms of the boundary layer variables. If $T(\varepsilon)$ denotes the regularizing transformation, which maps the original coordinates $(x)$ onto the boundary layer coordinates $(\xi)$, and $L_{\xi}(\varepsilon)$ is the differential expression in terms of the boundary layer variables, then

$$
L_{1,0}(\varepsilon)=T(\varepsilon)^{-1} L_{\xi}(0) T(\varepsilon), \quad L_{1,1}(\varepsilon)=T(\varepsilon)^{-1}\left(L_{\xi}(\varepsilon)-L_{\xi}(0)\right) T(\varepsilon) .
$$

Here, $L_{\xi}(0)$ denotes the formal limit of $L_{\xi}(\varepsilon)$ as $\varepsilon \downarrow 0$.

The convergence analysis of (2.35) is straightforward. The error at the $n$th step, $e_{i}^{(m, n)}=$ $u_{i}(\varepsilon)-u_{i}^{(m, n)}(\varepsilon)$, satisfies the homogeneous boundary value problem

$$
L_{i, 0}(\varepsilon) e_{i}^{(m, n)}=-L_{i, 1}(\varepsilon) e_{i}^{(m, n-1)} \text { on } \Omega_{i}, e_{i}^{(m, n)}=0 \text { on } \partial \Omega_{i}, i=0,1 .
$$

Assume that the expression $L_{i, 0}(\varepsilon)$ is invertible, and denote its inverse on $X_{0}\left(\Omega_{i}\right)$, the subspace of $X\left(\Omega_{i}\right)$ consisting of those elements that vanish on $\partial \Omega_{i}$, by $L_{i, 0}^{-1}(\varepsilon)$. Then,

$$
e_{i}^{(m, n)}=-L_{i, 0}^{-1}(\varepsilon) L_{i, 1}(\varepsilon) e_{i}^{(m, n-1)} .
$$

Let the amplification factor $\rho_{i}(\varepsilon)$ be defined by

$$
\rho_{i}(\varepsilon)=\lim \sup _{n \rightarrow \infty}\left(\left\|\left(L_{i, 0}^{-1}(\varepsilon) L_{i, 1}(\varepsilon)\right)^{n} e\right\|_{X\left(\Omega_{i}\right)} /\|e\|_{X\left(\Omega_{i}\right)}\right)^{1 / n}, i=0,1 .
$$

The definition is independent of the vector $e \in X_{0}\left(\Omega_{i}\right)$.

Lemma 3 If $\rho_{i}(\varepsilon)<1$, then

$$
\lim _{n \rightarrow \infty}\left\|u_{i}^{(m)}(\varepsilon)-u_{i}^{(m, n)}(\varepsilon)\right\|_{X\left(\Omega_{i}\right)}=0, i=0,1 .
$$

Proof. It follows from the definition (2.40) that

$$
\left\|u_{i}^{(m)}(\varepsilon)-u_{i}^{(m, n)}(\varepsilon)\right\|_{X\left(\Omega_{i}\right)} \leq\left(\rho_{i}(\varepsilon)\right)^{n}\left\|u_{i}^{(m)}(\varepsilon)-u_{i}^{(m, 0)}(\varepsilon)\right\|_{X\left(\Omega_{i}\right)}, i=0,1 .
$$

If $\rho_{i}(\varepsilon)<1$, the convergence follows. 
If $L_{i, 0}^{-1}(\varepsilon) L_{i, 1}(\varepsilon)$ is a bounded linear operator, then

$$
\rho_{i}(\varepsilon) \leq\left\|L_{i, 0}^{-1}(\varepsilon) L_{i, 1}(\varepsilon)\right\|_{\mathcal{L}\left(X\left(\Omega_{i}\right)\right)}
$$

so a sufficient (but not necessary) condition for convergence is that the operator norm of $L_{i, 0}^{-1}(\varepsilon) L_{i, 1}(\varepsilon)$ is less than one.

One realizes on a moment's reflection that Lemma 3 could never make sense in the continuous case, where the differential expressions lead to unbounded operators and the amplification factor is never going to be less than one. In the discrete case, however, Lemma 3 works out, because discrete approximations define bounded operators in finitedimensional vector spaces. The norms generally depend on the mesh width and grow beyond bounds as the mesh width goes to zero, but if the growth happens at a controlled rate as $\varepsilon \downarrow 0$, Lemma 3 provides a tool for the convergence analysis of the iterative procedure (2.35).

\subsection{Combining the Schwarz and Iterative Procedures}

We now combine the Schwarz alternating procedure and the iterative solution technique on each subdomain. Again, we take the continuous point of view.

Lemma 4 Suppose $L(\varepsilon)$ satisfies the maximum principle for every $\varepsilon \in\left(0, \varepsilon_{0}\right)$. If the propagation factor $\zeta$ of the Schwarz alternating procedure and the amplification factors $\rho_{i}$ $(i=0,1)$ of the iterative procedures satisfy the asymptotic order relations $\zeta(\varepsilon)=o(1)$ and $\rho_{i}(\varepsilon)=o(1)$ as $\varepsilon \downarrow 0$, then

$$
\left\|u_{i}(\varepsilon)-u_{i}^{\left(m, n_{i}\right)}(\varepsilon)\right\|_{X\left(\Omega_{i}\right)}=o\left(\zeta^{m}+\rho_{0}^{n_{0}}+\rho_{1}^{n_{1}}\right), i=0,1 .
$$

Proof. We use the abbreviations

$$
e_{i}^{(m, n)}=u_{i}(\varepsilon)-u_{i}^{(m, n)}(\varepsilon), n=0,1, \ldots ; \quad e_{i}^{(m)}=u_{i}(\varepsilon)-u_{i}^{(m)}(\varepsilon) ; \quad i=0,1 .
$$

From (2.42), we deduce the estimates

$$
\left\|e_{i}^{(m)}-e_{i}^{\left(m, n_{i}\right)}\right\|_{X\left(\Omega_{i}\right)} \leq \rho_{i}^{n_{i}}\left\|e_{i}^{(m)}-e_{i}^{(m, 0)}\right\|_{X\left(\Omega_{i}\right)}, i=0,1
$$

Hence,

$$
\left\|\gamma_{1} e_{0}^{\left(m, n_{0}\right)}\right\|_{X\left(\Gamma_{1}\right)} \leq\left\|\gamma_{1} e_{0}^{(m)}\right\|_{X\left(\Gamma_{1}\right)}+\rho_{0}^{n_{0}}\left\|e_{0}^{(m)}-e_{0}^{(m, 0)}\right\|_{X\left(\Omega_{0}\right)}
$$

We estimate the first term in the upper bound in terms of the solution on $\Omega_{1}$ obtained in the previous step in the Schwarz procedure,

$$
\left\|\gamma_{1} e_{0}^{(m)}\right\|_{X\left(\Gamma_{1}\right)} \leq \zeta_{0}\left\|\gamma_{0} e_{1}^{\left(m-1, n_{1}\right)}\right\|_{X\left(\Gamma_{0}\right)}
$$


A rough estimate of the second term is obtained by going back a whole step in the Schwarz procedure,

$$
\left\|e_{0}^{(m)}-e_{0}^{(m, 0)}\right\|_{X\left(\Omega_{0}\right)} \leq \zeta\left\|e_{0}^{(m-1)}\right\|_{X\left(\Omega_{0}\right)}+\rho_{0}^{n_{0}}\left\|e_{0}^{(m-1,0)}\right\|_{X\left(\Omega_{0}\right)} .
$$

This estimate shows that, as $\zeta=o(1)$ and $\rho_{0}=o(1)$ as $\varepsilon \downarrow 0$, it is certainly possible to achieve the inequality $\left\|e_{0}^{(m)}-e_{0}^{(m, 0)}\right\|_{X\left(\Omega_{0}\right)} \leq 1$ for $m=1,2, \ldots$; if necessary, we decrease $\varepsilon_{0}$. Thus, (2.45) reduces to

$$
\left\|\gamma_{1} e_{0}^{\left(m, n_{0}\right)}\right\|_{X\left(\Gamma_{1}\right)} \leq \zeta_{0}\left\|\gamma_{0} e_{1}^{\left(m-1, n_{1}\right)}\right\|_{X\left(\Gamma_{0}\right)}+\rho_{0}^{n_{0}}
$$

Similarly,

$$
\left\|\gamma_{0} e_{1}^{\left(m, n_{1}\right)}\right\|_{X\left(\Gamma_{0}\right)} \leq \zeta_{1}\left\|\gamma_{1} e_{0}^{\left(m, n_{0}\right)}\right\|_{X\left(\Gamma_{1}\right)}+\rho_{1}^{n_{1}}
$$

Combining (2.46) and (2.47), we obtain the estimate

$$
\left\|\gamma_{1} e_{0}^{\left(m, n_{0}\right)}\right\|_{X\left(\Gamma_{1}\right)} \leq \zeta\left\|\gamma_{1} e_{0}^{\left(m-1, n_{0}\right)}\right\|_{X\left(\Gamma_{1}\right)}+\zeta_{0} \rho_{1}^{n_{1}}+\rho_{0}^{n_{0}} .
$$

Repeated application of this inequality yields the estimate

$$
\left\|\gamma_{1} e_{0}^{\left(m, n_{0}\right)}\right\|_{X\left(\Gamma_{1}\right)} \leq \zeta^{m}\left\|\gamma_{1} e_{0}^{\left(0, n_{0}\right)}\right\|_{X\left(\Gamma_{1}\right)}+(1-\zeta)^{-1}\left(\zeta_{0} \rho_{1}^{n_{1}}+\rho_{0}^{n_{0}}\right) .
$$

Therefore,

$$
\left\|\gamma_{1} e_{0}^{\left(m, n_{0}\right)}\right\|_{X\left(\Gamma_{1}\right)}=o\left(\zeta^{m}+\rho_{0}^{n_{0}}+\rho_{1}^{n_{1}}\right) .
$$

By applying the maximum principle, we extend this asymptotic estimate to $\Omega_{0}$. The asymptotic estimate on $\Omega_{1}$ is obtained similarly.

The results of this section are summarized in the following theorem.

Theorem 2 (Convergence) The convergence of the Schwarz alternating procedure (2.23), combined with the iterative solution (2.35) on each subdomain, is optimal if the number of steps $n_{i}$ in (2.35) and the number of steps $m$ in (2.23) is chosen in such a way that $\rho_{i}^{n_{i}} \approx \rho^{n^{n}}$ for $i=0,1$ and $\zeta^{m}=O\left(\rho^{n}\right)$ as $\varepsilon \downarrow 0$. The convengence rate of the combined procedure depends on $\varepsilon$ and is asymptotically of the same order as $\rho$.

In summary, the method proposed here for the numerical solution of singular perturbation problems of the type (1.1) has two aspects. First, there is the domain decomposition and subsequent discretization, going from (1.1) to (2.14). Here, the issue is accuracy; it is addressed in Theorem 1. Then, there is the Schwarz alternating procedure and the iterative solution, going from (2.14) to (2.35). Here, the issue is convergence; it is addressed in Theorem 2. The success of the method depends on whether it is possible to choose the mesh widths as a function of $\varepsilon$ in such a way that both the accuracy and the convergence rate improve as $\varepsilon \downarrow 0$. In the following sections we show that this goal can indeed be accomplished for three second-order problems of increasing complexity. We restrict the discussion to two-dimensional problems (coordinates $x$ and $y$ ). 


\section{A Singularly Perturbed Zero-Order Equation}

We begin with the singular perturbation problem

$$
L(\varepsilon) u \equiv-\varepsilon \Delta u+q(x, y) u=f \text { on } \Omega, \quad u=g \text { on } \partial \Omega
$$

where the coefficient $q$ is strictly positive,

$$
q(x, y) \geq q_{0}>0,(x, y) \in \Omega .
$$

We take $\Omega=(0,1)^{2}$ and assume that there is a boundary layer (codimension one) along the left edge, $\Gamma=\{0\} \times(0,1)$, and no corner layers near the origin or the top left corner. The boundary layer coordinates are $\xi=\varepsilon^{-1 / 2} x$ and $\eta=y$.

We decompose $\Omega$ as in (2.5), with $\Omega_{0}=(b, 1) \times(0,1)$ and $\Omega_{1}=(0, a) \times(0,1)$, with $0<b<a \ll 1 ; a$ and $b$ depend on $\varepsilon$. The overlap region is $\Omega_{0} \cap \Omega_{1}=(b, a) \times(0,1)$. To obtain an asymptotically balanced scheme (Lemma 1 ), we require that $a$ and $b$ satisfy the order relations

$$
a(\varepsilon) \approx b(\varepsilon) \approx \varepsilon^{1 / 2} \log \left(\varepsilon^{-1}\right) .
$$

We impose regular rectangular grids on $\Omega_{0}$ and $\Omega_{1}$,

$$
\left.\left.\Omega_{h 0}=\left\{\left(1-b+i h_{0, x}, j h_{0, y}\right)\right): i, j=0, \ldots, N\right\}, \Omega_{h 1}=\left\{\left(i h_{1, x}, j h_{1, y}\right)\right): i, j=0, \ldots, N\right\} .
$$

The grids have the same number of mesh cells in the $x$ and $y$ directions, so the mesh widths are $h_{0, x}=(1-b) / N$ and $h_{0, y}=1 / N$ on $\Omega_{0}, h_{1, x}=a / N$ and $h_{1, y}=1 / N$ on $\Omega_{1}$. The boundary points are obtained if either $i$ or $j$ is equal to 0 or $N$. The composite grid is $\Omega_{h}$. Because the coarse grid must conform with the overlap region, and $\Omega_{h 1}$ is a refinement of $\Omega_{h 0}$ there, it must be the case that $a=b+k_{0} h_{0, x}$ and $a=b+k_{1} h_{1, x}$ for some pair of integers $\left(k_{0}, k_{1}\right)$. In terms of $N, k_{0}$, and $k_{1}$, we have

$$
a=\frac{k_{0} / N}{1-\left(1-k_{0} / N\right)\left(1-k_{1} / N\right)}, \quad b=\frac{\left(k_{0} / N\right)\left(1-k_{1} / N\right)}{1-\left(1-k_{0} / N\right)\left(1-k_{1} / N\right)} .
$$

A minimum overlap decomposition results if $k_{0}=1$.

The choice of a discretization procedure is open, except for the fact that the approximations must satisfy the maximum principle on each subdomain and that an estimate of the type (2.15) must hold. We choose the usual five-point stencil approximation of the Laplacian on each subdomain, which is second-order accurate in both directions. The estimate (2.15) holds with $k_{0}=k_{1}=2$ and $l_{0}=l_{1}=2$, and we conclude from (2.22) that a good asymptotic estimate of the truncation error is given by

$$
\left\|\left.u_{\Omega}(\varepsilon)\right|_{\Omega_{h}}-u(\varepsilon, h)\right\|_{X\left(\Omega_{h}\right)}=O\left(N^{-2} \log \left(\varepsilon^{-1}\right)\right) .
$$


It remains to fix the dependence of $N$ on $\varepsilon$. The choice is the result of a compromise, which seeks to maximize both the accuracy and the convergence rate. An appropriate choice for the present problem is

$$
N \approx\left(\varepsilon^{1 / 2} \log \left(\varepsilon^{-1}\right)\right)^{-1}
$$

Once this choice has been made, the grid $\Omega_{h}$ becomes connected asymptotically with $\varepsilon$. We emphasize this fact by writing $\Omega_{h(\varepsilon)}$. The asymptotic behavior of $h_{0}=\left(h_{0, x}, h_{0, y}\right)$ and $h_{1}=\left(h_{1, x}, h_{1, y}\right)$ is summarized by the expressions

$$
h_{0}(\varepsilon) \approx(h(\varepsilon), h(\varepsilon)), h_{1}(\varepsilon) \approx\left(\varepsilon^{1 / 2} h(\varepsilon), h(\varepsilon)\right)
$$

where $h=N^{-1}$ is a characteristic global mesh width,

$$
h(\varepsilon) \approx \varepsilon^{1 / 2} \log \left(\varepsilon^{-1}\right)
$$

The accuracy of the approximation obtained on the grid $\Omega_{h(\varepsilon)}$ is a function of $\varepsilon$ alone. In fact, we see from (3.6) that the truncation error decreases almost linearly as $\varepsilon \downarrow 0$,

$$
\left\|\left.u_{\Omega}(\varepsilon)\right|_{\Omega_{h(c)}}-u(\varepsilon, h(\varepsilon))\right\|_{X\left(\Omega_{h(c)}\right)}=O\left(\varepsilon \log \left(\varepsilon^{-1}\right)\right) .
$$

The asymptotic order of the propagation numbers of the Schwarz alternating procedure, $\zeta_{0}$ and $\zeta_{1}$, can be computed [25, Lemma 4]. With $a=b+h_{0, x}$ (minimum overlap, $k_{0}=1$ in $(3.5)$ ), we have $\zeta_{0} \approx \varepsilon$ and $\zeta_{1} \approx\left(\log \left(\varepsilon^{-1}\right)\right)^{-2}$, so the propagation factor of the Schwarz alternating method satisfies the estimate

$$
\zeta(\varepsilon)=O\left(\varepsilon\left(\log \left(\varepsilon^{-1}\right)\right)^{-1}\right)
$$

(Recall that we always lump all powers of the logarithm of $\varepsilon^{-1}$ into one.)

\subsection{Regular Domain}

On the regular domain, we use a cut-off function $\chi:\left(0, \varepsilon_{0}\right) \rightarrow X(\Omega)$, which is identically equal to one in the overlap region and zero one mesh cell beyond the overlap region,

$$
\chi(\varepsilon)(x, y)= \begin{cases}1 & \text { if } b \leq x \leq a=b+k_{0} h_{0, x} \\ 0 & \text { if } x \geq a+h_{0, x}=b+\left(k_{0}+1\right) h_{0, x}\end{cases}
$$

Following the definitions (2.34) and (2.36), we take $L(\varepsilon)=L_{0,0}(\varepsilon)+L_{0,1}(\varepsilon)$, where

$$
L_{0,0}(\varepsilon) u=-\varepsilon \chi(\varepsilon) \Delta u+q(x, y) u, \quad L_{0,1}(\varepsilon) u=-\varepsilon(1-\chi(\varepsilon)) \Delta u .
$$

Thus, beyond the overlap region, the $x$ and $y$ derivatives are both treated explicitly. The discrete approximations are

$$
\left(L_{h 0,0}(\varepsilon) u\right)_{i, j}= \begin{cases}-\varepsilon\left(\Delta_{h 0} u\right)_{i, j}+q_{h 0 ; i, j} u_{i, j}, & \text { if } i=1, \ldots, k_{0} \\ q_{h 0 ; i, j} u_{i, j} & \text { if } i=k_{0}+1, \ldots, N-1,\end{cases}
$$




$$
\left(L_{h 0,1}(\varepsilon) u\right)_{i, j}= \begin{cases}0, & \text { if } i=1, \ldots, k_{0}, \\ -\varepsilon\left(\Delta_{h 0} u\right)_{i, j}, & \text { if } i=k_{0}+1, \ldots, N-1,\end{cases}
$$

where $\Delta_{h 0}$ is the five-point stencil approximation to the Laplacian on the grid $\Omega_{h 0}$,

$$
\left(\Delta_{h 0} u\right)_{i, j}=\frac{u_{i+1, j}-2 u_{i, j}+u_{i-1, j}}{h_{0, x}^{2}}+\frac{u_{i, j+1}-2 u_{i, j}+u_{i, j-1}}{h_{0, y}^{2}}
$$

We proceed to estimate the amplification factor $\rho_{0}(\varepsilon) ; \mathrm{cf} .(2.40)$. The differential expression $L_{0,0}(\varepsilon)$ is a small perturbation of the zero-order expression $q$ (i.e., multiplication by the local value of $q$ ), which is positive everywhere. Hence, the inverse $L_{0,0}^{-1}(\varepsilon)$ exists and is asymptotically bounded in $\mathcal{L}\left(X\left(\Omega_{0}\right)\right)$ by a constant that does not depend on $\varepsilon$. This property carries over to its discrete analog. The expression $L_{0,1}(\varepsilon)$ is certainly unbounded. However, its discrete analog, $L_{h 0,1}(\varepsilon)$, is bounded in $\mathcal{L}\left(X\left(\Omega_{h 0}\right)\right)$; in fact, its norm is $4 \varepsilon h_{0, x}^{-2}+4 \varepsilon h_{0, y}^{-2}$. Consequently, $L_{h 0,0}^{-1}(\varepsilon) L_{h 0,1}(\varepsilon)$ is an element of $\mathcal{L}\left(X\left(\Omega_{h 0}\right)\right)$, and its norm is bounded by a constant multiple of $\varepsilon h_{0, x}^{-2}+\varepsilon h_{0, y}^{-2}$. The latter quantity is of order $\left(\log \left(\varepsilon^{-1}\right)\right)^{-1}$ on $\Omega_{h(\varepsilon)}$. Hence,

$$
\rho_{0}(\varepsilon)=O\left(\left(\log \left(\varepsilon^{-1}\right)\right)^{-1}\right) \text { on } \Omega_{h(\varepsilon)}
$$

so convergence is assured for all sufficiently small $\varepsilon$.

We observe that we can relax the iterative procedure without jeopardizing its convergence. Instead of (3.14) and (3.15), we may take

$$
\begin{aligned}
& \left(L_{h 0,0}(\varepsilon) u\right)_{i, j}= \begin{cases}-\varepsilon\left(\Delta_{h 0} u\right)_{i, j}+q_{h 0 ; i, j} u_{i, j}, & \text { if } i=1, \ldots, k_{0}, \\
\left(q_{h 0 ; i, j}+r(\varepsilon)\right) u_{i, j} & \text { if } i=k_{0}+1, \ldots, N-1,\end{cases} \\
& \left(L_{h 0,1}(\varepsilon) u\right)_{i, j}= \begin{cases}0, & \text { if } i=1, \ldots, k_{0}, \\
-\varepsilon\left(\Delta_{h 0} u\right)_{i, j}-r(\varepsilon) u_{i, j}, & \text { if } i=k_{0}+1, \ldots, N-1,\end{cases}
\end{aligned}
$$

where the $\varepsilon$-dependent relaxation factor is

$$
r(\varepsilon)=\frac{2 \varepsilon}{h_{0, x}^{2}}+\frac{2 \varepsilon}{h_{0, y}^{2}} .
$$

The amplification factor, which is $\rho_{0}=r\left(q_{0}+r\right)^{-1}$, satisfies the same estimate, (3.17).

\subsection{Boundary Layer}

Following the definitions (2.34) and (2.37), we base the iterative solution in the boundary layer on the splitting $L(\varepsilon)=L_{1,0}(\varepsilon)+L_{1,1}(\varepsilon)$, where

$$
L_{1,0}(\varepsilon) u=-\varepsilon \partial_{x}^{2} u+q(x, y) u, \quad L_{1,1}(\varepsilon) u=-\varepsilon \partial_{y}^{2} u .
$$


Thus, the derivatives with respect to $x$ are treated implicitly, those with respect to $y$ explicitly. The discrete approximations are

$$
\begin{gathered}
\left(L_{h 1,0}(\varepsilon) u\right)_{i, j}=-\varepsilon \frac{u_{i+1, j}-2 u_{i, j}+u_{i-1, j}}{h_{1, x}^{2}}+q_{h 1 ; i, j} u_{i, j} \\
\left(L_{h 1,1}(\varepsilon) u\right)_{i, j}=-\varepsilon \frac{u_{i, j+1}-2 u_{i, j}+u_{i, j-1}}{h_{1, y}^{2}}
\end{gathered}
$$

We proceed to estimate the amplification factor $\rho_{1}(\varepsilon)$; cf. $(2.40)$. We claim that $L_{1,0}^{-1}(\varepsilon)$ is bounded in norm by $a(\varepsilon) \varepsilon^{-1 / 2}$. In the boundary layer, the appropriate variables are $\xi=$ $\varepsilon^{-1 / 2} x$ and $\eta=y$; they extend over the rectangle $\left(0, a(\varepsilon) \varepsilon^{-1 / 2}\right) \times(0,1)$. Upon transformation to the boundary layer variables, $L_{1,0}(\varepsilon)$ reduces to the differential expression $-\partial_{\xi}^{2}+q$. The Green's function for this Sturm-Liouville expression, subject to homogeneous (Dirichlet) boundary conditions, is uniformly continuous, so the corresponding linear integral operator considered on the space of continuous functions is bounded in norm by $a(\varepsilon) \varepsilon^{-1 / 2}$. It follows that $L_{1,0}^{-1}(\varepsilon)$ in $\mathcal{L}\left(X\left(\Omega_{1}\right)\right)$ is also bounded in norm by $a(\varepsilon) \varepsilon^{-1 / 2}$. This property carries over to the discrete analog, $L_{h 1,0}^{-1}(\varepsilon)$. Of course, $L_{1,1}(\varepsilon)$ is unbounded, but its discrete analog, $L_{h 1,1}(\varepsilon)$, is bounded; in fact, its norm in $\mathcal{L}\left(X\left(\Omega_{h 1}\right)\right)$ is $4 \varepsilon h_{1, y}^{-2}$. Consequently, $L_{h 1,0}^{-1}(\varepsilon) L_{h 1,1}(\varepsilon)$ is en element of $\mathcal{L}\left(X\left(\Omega_{h 1}\right)\right)$, and its norm is bounded by a constant multiple of $a(\varepsilon) \varepsilon^{1 / 2} h_{1, y}^{-2}$. The latter quantity is of order $\left(\log \left(\varepsilon^{-1}\right)\right)^{-1}$ on $\Omega_{h(\varepsilon)}$. Hence,

$$
\rho_{1}(\varepsilon)=O\left(\left(\log \left(\varepsilon^{-1}\right)\right)^{-1}\right) \text { on } \Omega_{h(\varepsilon)}
$$

so convergence is again assured for all sufficiently small $\varepsilon$.

As in the regular domain, we can relax the iterative procedure without jeopardizing its convergence. Instead of (3.22) and (3.23), we may take

$$
\begin{gathered}
\left(L_{h 1,0}(\varepsilon) u\right)_{i, j}=-\varepsilon \frac{u_{i+1, j}-2 u_{i, j}+u_{i-1, j}}{h_{1, x}^{2}}+\left(q_{h 1 ; i, j}+r(\varepsilon)\right) u_{i, j}, \\
\left(L_{h 1,1}(\varepsilon) u\right)_{i, j}=-\varepsilon \frac{u_{i, j+1}-2 u_{i, j}+u_{i, j-1}}{h_{1, y}^{2}}-r(\varepsilon) u_{i, j}
\end{gathered}
$$

where $r$ is an $\varepsilon$-dependent relaxation factor,

$$
r(\varepsilon)=\frac{2 \varepsilon}{h_{1, y}^{2}}
$$

The amplification factor, which is $\rho_{1}=r\left(q_{0}+r\right)^{-1}$, satisfies the same estimate, (3.24).

Given the asymptotic results (3.11), (3.17), and (3.24), we conclude from Theorem 2 that the Schwarz alternating procedure, combined with the iterative solution techniques, converges at a rate of $\left(\log \left(\varepsilon^{-1}\right)\right)^{-1}$. 


\subsection{General Configurations}

If $\Omega$ is more generally a simply connected domain with smooth boundary, and a boundary layer is adjacent to some part $\Gamma$ of the boundary, much of the preceding theory still applies. In the boundary layer, we introduce a local coordinate system with coordinates $s$ and $t$, such that the Laplacian is represented in divergence form,

$$
\Delta_{s, t} u=(a(s, t))^{-1} \partial_{s} a(s, t) \partial_{s} u+\partial_{t}(a(s, t))^{-1} \partial_{t} u ;
$$

$a$ is a known function of $s$ and $t$, which is strictly positive and bounded. Such a coordinate system certainly exists; see [30, Section IV.8.2]. The regularizing transformation stretches the coordinate in the $s$ direction by a factor $\varepsilon^{-1 / 2}$. Instead of (3.22) and (3.23), we have the more complicated expressions

$$
\begin{gathered}
\left(L_{h 1,0}(\varepsilon) u\right)_{i, j}=-\varepsilon a_{i, j}^{-1} \frac{a_{i+1 / 2, j}\left(\delta_{s}^{+} u\right)_{i, j}-a_{i-1 / 2, j}\left(\delta_{s}^{-} u\right)_{i, j}}{h_{1, s}}+q_{h 1 ; i, j} u_{i, j}, \\
\left(L_{h 1,1}(\varepsilon) u\right)_{i, j}=-\varepsilon \frac{a_{i, j+1 / 2}^{-1}\left(\delta_{t}^{+} u\right)_{i, j}-a_{i, j-1 / 2}^{-1}\left(\delta_{t}^{-} u\right)_{i, j}}{h_{1, t}}
\end{gathered}
$$

where $a_{i+1 / 2, j}=\frac{1}{2}\left(a_{i, j}+a_{i+1, j}\right), \delta_{s}^{ \pm}$are the forward and backward differences in the $s$ direction,

$$
\left(\delta_{s}^{+} u\right)_{i, j}=\frac{u_{i+1, j}-u_{i, j}}{h_{1, s}},\left(\delta_{s}^{-} u\right)_{i, j}=\frac{u_{i, j}-u_{i-1, j}}{h_{1, s}}
$$

and similarly in the $t$ direction. In the relaxed scheme, one takes a variable relaxation factor, $r_{i, j}=\varepsilon\left(a_{i, j+1 / 2}^{-1}+a_{i, j-1 / 2}^{-1}\right) h_{1, t}^{-2}$.

\section{A Singularly Perturbed Convection Problem}

Next, we consider the singular perturbation problem

$$
-\varepsilon \Delta u+p(x, y) \partial_{x} u=f \text { on } \Omega, u=g \text { on } \partial \Omega,
$$

where $p$ is strictly positive,

$$
p(x, y) \geq p_{0}>0,(x, y) \in \Omega .
$$

We assume that $p$ is differentiable with respect to $x$, with a uniformly bounded derivative on $\bar{\Omega}$. Again, we take $\Omega=(0,1)^{2}$. The unperturbed equation prescribes the rate of change of the solution in the $x$ direction. If the boundary data are incompatible with this rate of change, a boundary layer will develop. If the coefficient $p$ is positive, the boundary layer will be adjacent to the right edge, so $\Gamma=\{1\} \times(0,1)$. Again, we assume that there are no corner layers. The boundary layer coordinates are $\xi=\varepsilon^{-1}(1-x)$ and $\eta=y$. 
We decompose $\Omega$ into two overlapping subdomains, $\Omega_{0}=(0,1-b) \times(0,1)$ and $\Omega_{1}=$ $(1-a, 1) \times(0,1)$, with $0<b<a \ll 1$. The overlap region is $\Omega_{0} \cap \Omega_{1}=(1-a, 1-b) \times(0,1)$. To obtain an asymptotically balanced scheme (Lemma 1), we require that $a$ and $b$ satisfy the asymptotic order relations

$$
a(\varepsilon) \approx b(\varepsilon) \approx \varepsilon \log \left(\varepsilon^{-1}\right) .
$$

We impose rectangular grids on $\Omega_{0}$ and $\Omega_{1}$,

$$
\begin{aligned}
& \left.\Omega_{h 0}=\left\{\left(i h_{0, x}, j h_{0, y}\right)\right): i=0, \ldots, N_{x} ; j=0, \ldots, N_{y}\right\}, \\
& \Omega_{h 1}=\left\{\left(1-a+i h_{1, x}, j h_{1, y}\right): i=0, \ldots, N_{x} ; j=0, \ldots, N_{y}\right\} .
\end{aligned}
$$

The grids have a different number of mesh cells in the $x$ and $y$ directions, and the mesh widths are $h_{0, x}=(1-b) / N_{x}$ and $h_{0, y}=1 / N_{y}$ on $\Omega_{0}, h_{1, x}=a / N_{x}$ and $h_{1, y}=1 / N_{y}$ on $\Omega_{1}$. We approximate the Laplacian by the usual five-point stencil and the derivative in the $x$ direction by backward differences. (If $p$ is negative, the boundary layer is along the left edge of the domain, and one takes the forward-difference approximation.) The approximation is first-order accurate in the $x$ direction and second-order accurate in the $y$ direction. According to (2.22), the truncation error satisfies the estimate

$$
\left\|\left.u_{\Omega}(\varepsilon)\right|_{\Omega_{h}}-u(\varepsilon, h)\right\|_{X\left(\Omega_{h}\right)}=O\left(N_{x}^{-1} \log \left(\varepsilon^{-1}\right)+N_{y}^{-2} \log \left(\varepsilon^{-1}\right)\right) .
$$

With the choice

$$
N_{x} \approx\left(\varepsilon \log \left(\varepsilon^{-1}\right)\right)^{-1}, \quad N_{y} \approx\left(\varepsilon^{1 / 2} \log \left(\varepsilon^{-1}\right)\right)^{-1},
$$

we tie the grid $\Omega_{h}$ in with $\varepsilon$. We write $\Omega_{h(\varepsilon)}$ and summarize the asymptotic behavior of the mesh widths by

$$
h_{0}(\varepsilon) \approx\left(h_{x}(\varepsilon), h_{y}(\varepsilon)\right), h_{1}(\varepsilon) \approx\left(\varepsilon h_{x}(\varepsilon), h_{y}(\varepsilon)\right),
$$

where $h_{x}=N_{x}^{-1}$ and $h_{y}=N_{y}^{-1}$ are characteristic global mesh widths,

$$
h_{x}(\varepsilon) \approx \varepsilon \log \left(\varepsilon^{-1}\right), h_{y}(\varepsilon) \approx \varepsilon^{1 / 2} \log \left(\varepsilon^{-1}\right) .
$$

On $\Omega_{h(\varepsilon)}$, the truncation error decreases almost linearly as $\varepsilon \downarrow 0$,

$$
\left\|\left.u_{\Omega}(\varepsilon)\right|_{\Omega_{h(c)}}-u(\varepsilon, h(\varepsilon))\right\|_{X\left(\Omega_{h(\varepsilon)}\right)}=O\left(\varepsilon \log \left(\varepsilon^{-1}\right)\right) .
$$

The asymptotic order of the propagation numbers of the Schwarz alternating procedure can be computed [25, Lemma 5]. With the minimum overlap, we have $\zeta_{0} \approx 1$ and $\zeta_{1} \approx$ $\left(\log \left(\varepsilon^{-1}\right)\right)^{-1}$, so

$$
\zeta(\varepsilon) \approx\left(\log \left(\varepsilon^{-1}\right)\right)^{-1}
$$




\subsection{Regular Domain}

On the regular domain, we use a cut-off function $\chi$, which is identically one in the overlap region and zero one mesh cell beyond the overlap region,

$$
\chi(\varepsilon)(x, y)= \begin{cases}0 & \text { if } x \leq 1-a-h_{0, x}=\left(N_{x}-k_{0}-1\right) h_{0, x} \\ 1 & \text { if } 1-a=\left(N_{x}-k_{0}\right) h_{0, x} \leq x \leq 1-b .\end{cases}
$$

The iterative procedure is based on the splitting $L(\varepsilon)=L_{0,0}(\varepsilon)+L_{0,1}(\varepsilon)$, where

$$
L_{0,0} u=-\varepsilon \chi(\varepsilon) \Delta u+p(x, y) \partial_{x} u, \quad L_{0,1} u=-\varepsilon(1-\chi(\varepsilon)) \Delta u .
$$

The discrete approximations are

$$
\begin{gathered}
\left(L_{h 0,0}(\varepsilon) u\right)_{i, j}= \begin{cases}p_{h 0 ; i, j}\left(\delta_{h 0, x}^{-} u\right)_{i, j}, & i=1, \ldots, N_{x}-k_{0}-1, \\
p_{h 0 ; i, j}\left(\delta_{h 0, x}^{-} u\right)_{i, j}-\varepsilon\left(\Delta_{h 0} u\right)_{i, j}, & i=N_{x}-k_{0}, \ldots, N_{x}-1,\end{cases} \\
\left(L_{h 0,1}(\varepsilon) u\right)_{i, j}= \begin{cases}-\varepsilon\left(\Delta_{h 0} u\right)_{i, j}, & i=1, \ldots, N_{x}-k_{0}-1, \\
0 & i=N_{x}-k_{0}, \ldots, N_{x}-1,\end{cases}
\end{gathered}
$$

where

$$
\begin{gathered}
\left(\Delta_{h 0} u\right)_{i, j}=\frac{u_{i+1, j}-2 u_{i, j}+u_{i-1, j}}{h_{0, x}^{2}}+\frac{u_{i, j+1}-2 u_{i, j}+u_{i, j-1}}{h_{0, y}^{2}}, \\
\left(\delta_{h 0, x}^{-} u\right)_{i, j}=\frac{u_{i, j}-u_{i-1, j}}{h_{0, x}} .
\end{gathered}
$$

We proceed to estimate the amplification factor. The differential expression $L_{0,0}(\varepsilon)$ is a small perturbation of the first-order expression $p \partial_{x}$, where $p$ is positive everywhere. Its inverse on the subspace $X_{0}\left(\Omega_{0}\right)$ of $X\left(\Omega_{0}\right)$ is a simple integral, so

$$
\left(L_{0,0}^{-1}(\varepsilon) L_{0,1}(\varepsilon) e\right)(x, y)=-\varepsilon \int_{0}^{x} p^{-1}(z, y)\left(\partial_{z}^{2} e+\partial_{y}^{2} e\right)(z, y) d z
$$

Upon integration by parts, we obtain

$$
\begin{aligned}
\left(L_{0,0}^{-1}(\varepsilon) L_{0,1}(\varepsilon) e\right)(x, y)= & -\varepsilon\left\{\left[p^{-1} \partial_{z} e(z, y)\right]_{z=0}^{z=x}-\int_{0}^{x} \partial_{z} p^{-1}(z, y) \partial_{z} e(z, y) d z\right\} \\
& -\varepsilon \int_{0}^{x} p^{-1}(z, y) \partial_{y}^{2} e(z, y) d z
\end{aligned}
$$

Here, $p^{-1}$ and its derivative with respect to the first argument are uniformly bounded.

The expression in the right member is certainly unbounded. But consider its discrete analog. The integrals are replaced by sums, and derivatives by finite differences; function evaluations take place on the grid. On each subinterval, we can estimate the derivate $\partial_{z} e$ 
by $2\|e\| h_{0, x}^{-1}$ and the derivative $\partial_{y}^{2} e$ by $4\|e\| h_{0, y}^{-2}$. Thus, there exists a positive constant $C$ such that

$$
\left|\left(L_{h 0,0}^{-1}(\varepsilon) L_{h 0,1}(\varepsilon) e\right)_{i, j}\right| \leq C\left(\frac{\varepsilon}{h_{0, x}}+\frac{\varepsilon}{h_{0, y}^{2}}\right)\|e\|_{X\left(\Omega_{h 0}\right)} .
$$

The estimate shows that $L_{h 0,0}^{-1}(\varepsilon) L_{h 0,1}(\varepsilon)$ is a bounded linear operator on $X\left(\Omega_{h 0}\right)$ and that its norm is bounded by a constant multiple of $\varepsilon h_{0, x}^{-1}+\varepsilon h_{0, y}^{-2}$. The bound is of order $\left(\log \left(\varepsilon^{-1}\right)\right)^{-1}$ on $\Omega_{h(\varepsilon)}$, so

$$
\rho_{0}(\varepsilon)=O\left(\left(\log \left(\varepsilon^{-1}\right)\right)^{-1}\right) \text { on } \Omega_{h(\varepsilon)},
$$

and convergence is assured for all sufficiently small $\varepsilon$.

The iterative procedure can be relaxed,

$$
\begin{aligned}
& \left(L_{h 0,0}(\varepsilon) u\right)_{i, j}= \begin{cases}\left(p_{h 0 ; i, j}+r(\varepsilon)\right)\left(\delta_{h 0, x}^{-} u\right)_{i, j}, & i=1, \ldots, N_{x}-k_{0}-1, \\
p_{h 0 ; i, j}\left(\delta_{h 0, x}^{-} u\right)_{i, j}-\varepsilon\left(\Delta_{h 0} u\right)_{i, j}, & i=N_{x}-k_{0}, \ldots, N_{x}-1,\end{cases} \\
& \left(L_{h 0,1}(\varepsilon) u\right)_{i, j}= \begin{cases}-\varepsilon\left(\Delta_{h 0} u\right)_{i, j}-r(\varepsilon)\left(\delta_{h 0, x}^{-} u\right)_{i, j}, & i=1, \ldots, N_{x}-k_{0}-1, \\
0 & i=N_{x}-k_{0}, \ldots, N_{x}-1,\end{cases}
\end{aligned}
$$

where

$$
r(\varepsilon)=\frac{\varepsilon}{h_{0, x}} .
$$

The amplification factor satisfies the same estimate, (4.20).

\subsection{Boundary Layer}

For the iterative procedure, we take $L(\varepsilon)=\varepsilon^{-1}\left(L_{1,0}(\varepsilon)+L_{1,1}(\varepsilon)\right)$, where

$$
L_{1,0}(\varepsilon) u=-\varepsilon^{2} \partial_{x}^{2} u+\varepsilon p(x, y) \partial_{x} u, \quad L_{1,1}(\varepsilon) u=-\varepsilon^{2} \partial_{y}^{2} u .
$$

The extra factor $\varepsilon$ is brought in for convenience. The discrete approximations are

$$
\begin{gathered}
\left(L_{h 1,0}(\varepsilon) u\right)_{i, j}=-\varepsilon^{2} \frac{u_{i+1, j}-2 u_{i, j}+u_{i-1, j}}{h_{1, x}^{2}}+\varepsilon p_{h 1 ; i, j} \frac{u_{i, j}-u_{i-1, j}}{h_{1, x}}, \\
\left(L_{h 1,1}(\varepsilon) u\right)_{i, j}=-\varepsilon^{2} \frac{u_{i, j+1}-2 u_{i, j}+u_{i, j-1}}{h_{1, y}^{2}}
\end{gathered}
$$

We use an energy argument to estimate the amplification factor. We first analyze the semidiscrete case, where $x$ ranges over the interval $(1-a(\varepsilon), 1)$ and $y$ is discrete. In the $n$th step of the iterative procedure, the error $e_{j}^{(n)}=u_{j}^{(m)}-u_{j}^{(m, n)}$ at $y=y_{j}$ satisfies the equation

$$
-\varepsilon^{2} \partial_{x}^{2} e_{j}^{(n)}+\varepsilon p_{j}(x) \partial_{x} e_{j}^{(n)}=\varepsilon^{2} \frac{e_{j+1}^{(n-1)}-2 e_{j}^{(n-1)}+e_{j-1}^{(n-1)}}{h_{1, y}^{2}}
$$


on the interval $(1-a(\varepsilon), 1)$ and zero boundary conditions at the end points. (The index $m$ refers to the Schwarz step.) When we take the $L^{2}$-inner product over $(1-a(\varepsilon), 1)$ of both sides of the differential equation with $e_{j}^{(n)}$, integrate by parts, and use the identities $-\left(e, \partial_{x}^{2} e\right)=\left(\partial_{x} e, \partial_{x} e\right)$ and $\left(e, p_{j} \partial_{x} e\right)=-\frac{1}{2}\left(e, e \partial_{x} p_{j}\right)$ with $e=e_{j}^{(n)}$, we obtain the identity

$$
\varepsilon^{2}\left(\partial_{x} e_{j}^{(n)}, \partial_{x} e_{j}^{(n)}\right)-\frac{1}{2} \varepsilon\left(e_{j}^{(n)}, e_{j}^{(n)} \partial_{x} p_{j}\right)=\varepsilon^{2} h_{1, y}^{-2}\left(e_{j}^{(n)}, e_{j+1}^{(n-1)}-2 e_{j}^{(n-1)}+e_{j-1}^{(n-1)}\right) .
$$

We first estimate the quantity in the left member. (We drop the subscript $j$ and the superscript ( $n$ ) on $e$ temporarily.) The function $\partial_{x} p$ is uniformly bounded, so there exists a positive constant $C$ such that $\left|\left(e, e \partial_{x} p_{j}\right)\right| \leq C(e, e)$. Using the inequality

$$
(e, e) \leq a(\varepsilon)^{2}\left(\partial_{x} e, \partial_{x} e\right)
$$

we conclude that $\left|\left(e, e \partial_{x} p_{j}\right)\right| \leq C a(\varepsilon)^{2}\left(\partial_{x} e, \partial_{x} e\right)$. But $a(\varepsilon) \approx \varepsilon \log \left(\varepsilon^{-1}\right)$, so we may assume, without loss of generality, that $C a(\varepsilon)^{2} \leq \varepsilon$ for all $\varepsilon$; hence, $\left|\left(e, e \partial_{x} p_{j}\right)\right| \leq \varepsilon\left(\partial_{x} e, \partial_{x} e\right)$. This result leads to the following estimate of the quantity in the left member of (4.28),

$$
\left|\varepsilon^{2}\left(\partial_{x} e_{j}^{(n)}, \partial_{x} e_{j}^{(n)}\right)-\frac{1}{2} \varepsilon\left(e_{j}^{(n)}, e_{j}^{(n)} \partial_{x} p_{j}\right)\right| \geq \frac{1}{2} \varepsilon^{2}\left(\partial_{x} e_{j}^{(n)}, \partial_{x} e_{j}^{(n)}\right) .
$$

Next, we proceed to the right member of (4.28). Using the Cauchy-Schwarz inequality and the estimate (4.29) with $e=e_{j}^{(n)}$, we readily obtain a uniform bound for the inner product,

$$
\left|\left(e_{j}^{(n)}, e_{j+1}^{(n-1)}-2 e_{j}^{(n-1)}+e_{j-1}^{(n-1)}\right)\right| \leq 4 a(\varepsilon)\left\|\partial_{x} e^{(n)}\right\|_{L^{2}\left(\Omega_{1}\right)}\left\|e^{(n-1)}\right\|_{L^{2}\left(\Omega_{1}\right)} .
$$

Here, we have introduced the abbreviation

$$
\|f\|_{L^{2}\left(\Omega_{1}\right)}=\sup \left\{\left(f_{j}, f_{j}\right)^{1 / 2}: j=0, \ldots, N_{y}\right\} .
$$

Combining the inequalities (4.30) and (4.31) with (4.28), we find that

$$
\frac{1}{2}\left(\partial_{x} e_{j}^{(n)}, \partial_{x} e_{j}^{(n)}\right) \leq 4 a(\varepsilon) h_{1, y}^{-2}\left\|\partial_{x} e^{(n)}\right\|_{L^{2}\left(\Omega_{1}\right)}\left\|e^{(n-1)}\right\|_{L^{2}\left(\Omega_{1}\right)} .
$$

Taking the supremum over all $j$, we obtain the desired energy estimate,

$$
\frac{1}{2}\left\|\partial_{x} e^{(n)}\right\|_{L^{2}\left(\Omega_{1}\right)} \leq 4 a(\varepsilon) h_{1, y}^{-2}\left\|e^{(n-1)}\right\|_{L^{2}\left(\Omega_{1}\right)} \text {. }
$$

Since the errors and their partial derivatives are uniformly bounded, the energy estimate translates directly into an inequality involving the sup norms; in fact, for any $f$ we have

$$
\|f\|_{L^{2}\left(\Omega_{1}\right)} \leq a(\varepsilon)^{1 / 2}\|f\|_{X\left(\Omega_{3}\right)} \leq a(\varepsilon)\left\|\partial_{x} f\right\|_{L^{2}\left(\Omega_{1}\right)},
$$

so

$$
\left\|e^{(n)}\right\|_{X\left(\Omega_{1}\right)} \leq 8 a(\varepsilon)^{2} h_{1, y}^{-2}\left\|e^{(n-1)}\right\|_{X\left(\Omega_{1}\right)}
$$

This result carries over to the discrete case. It shows that the amplification factor is bounded by a constant multiple of $a(\varepsilon)^{2} h_{1, y}^{-2}$. The latter quantity is of order $\varepsilon\left(\log \left(\varepsilon^{-1}\right)\right)^{-1}$ on $\Omega_{h(\varepsilon)}$. Hence,

$$
\rho_{1}(\varepsilon)=O\left(\varepsilon \log \left(\varepsilon^{-1}\right)\right) \text { on } \Omega_{h(\varepsilon)} \text {. }
$$

Given the results $(4.10),(4.20)$, and (4.37), we conclude from Theorem 2 that the method converges and that the convergence rate is of the order of $\left(\log \left(\varepsilon^{-1}\right)\right)^{-1}$ as $\varepsilon \downarrow 0$. 


\subsection{General Configurations}

The preceding arguments generalize to the case where $\Omega$ is a simply connected domain with smooth boundary. Let $\mathbf{n}$ denote the unit vector normal to $\partial \Omega$ oriented toward the exterior of $\Omega$, and let $e_{x}$ denote the unit vector in the direction of increasing $x$. We use the symbols $\partial_{-} \Omega, \partial_{0} \Omega$, and $\partial_{+} \Omega$ to denote the part of $\partial \Omega$, where $\mathbf{n} \cdot \mathbf{e}_{x}$ is negative, zero, and positive, respectively. Because $\Omega$ is simply connected, $\partial \Omega$ is the union of these three disjoint sets. We assume that the singular part of the boundary, $\Gamma$, is a subset of $\partial_{+} \Omega$ and that $\Gamma$ is described by an equation of the form $x=\phi(y)$, where $\phi$ is smooth.

As usual, we decompose $\Omega$ into two overlapping subdomains, $\Omega_{0}$ and $\Omega_{1}$, where the latter covers the boundary layer. To obtain a balanced scheme, we choose the thickness of $\Omega_{1}$ to be asymptotically of the order of $\varepsilon \log \left(\varepsilon^{-1}\right)$.

In the boundary layer, we introduce nonorthogonal local coordinates $s$ and $t$,

$$
s=\phi(y)-x, t=\int_{0}^{y}\left(1+\left(\phi^{\prime}(\eta)\right)^{2}\right)^{1 / 2} d \eta .
$$

The inverse transformation is $x=-s+\phi(\psi(t)), y=\psi(t)$. In terms of the new coordinates, the differential equation in (4.1) assumes the form

$$
-\varepsilon\left[c_{20} \partial_{s}^{2}+c_{11} \partial_{s} \partial_{t}+c_{02} \partial_{t}^{2}+c_{10} \partial_{s}+c_{01} \partial_{t}\right] u-p \partial_{s} u=f
$$

The coefficients $c_{20}, \ldots$ are known; they depend on $t$ only, $c_{20}=c_{02}=\left(1+\left(\phi^{\prime}\right)^{2}\right), c_{11}=$ $2 \phi^{\prime}\left(1+\left(\phi^{\prime}\right)^{1 / 2}\right), c_{10}=\phi^{\prime \prime}$, and $c_{01}=\phi^{\prime} \phi^{\prime \prime}\left(1+\left(\phi^{\prime}\right)^{2}\right)^{-1 / 2}$. The regularizing transformation stretches the $s$ coordinate by a factor $\varepsilon^{-1}$.

Instead of (4.24), we have

$$
\begin{gathered}
L_{1,0}(\varepsilon) u=-\varepsilon^{2}\left(c_{20} \partial_{s}^{2} u+c_{10} \partial_{s} u\right)+\varepsilon p(s, t) \partial_{s} u, \\
L_{1,1}(\varepsilon) u=-\varepsilon^{2}\left(c_{11} \partial_{t} \partial_{s} u+c_{02} \partial_{t}^{2} u+c_{01} \partial_{t} u\right)
\end{gathered}
$$

and instead of (4.25) and (4.26),

$$
\begin{gathered}
\left(L_{h 1,0}(\varepsilon) u\right)_{i, j}=-\varepsilon^{2}\left[c_{20 ; j} \frac{u_{i+1, j}-2 u_{i, j}+u_{i-1, j}}{h_{1, s}^{2}}+c_{10 ; j} \frac{u_{i, j}-u_{i-1, j}}{h_{1, s}}\right]+\varepsilon p_{h 1 ; i, j} \frac{u_{i, j}-u_{i-1, j}}{h_{1, s}}, \\
\left(L_{h 1,1}(\varepsilon) u\right)_{i, j}=-\varepsilon^{2}\left[c_{11 ; j}\left(\frac{u_{i, j+1}-u_{i-1, j+1}}{2 h_{1, s} h_{1, t}}-\frac{u_{i, j-1}-u_{i-1, j-1}}{2 h_{1, s} h_{1, t}}\right)\right. \\
\left.+c_{02 ; j} \frac{u_{i, j+1}-2 u_{i, j}+u_{i, j-1}}{h_{1, t}^{2}}+c_{01 ; j} \frac{u_{i, j+1}-u_{i, j-1}}{2 h_{1, t}}\right] .
\end{gathered}
$$

The approximation is first-order accurate in $s$, second-order accurate in $t$. 
The amplification factor can be found with the same type of argument from an energy estimate, but there is a complication because of the occurrence of the $s$-derivative in $L_{1,1}$. Again, it is convenient to analyze the semi-discrete case. Instead of (4.28), we have the more complicated identity

$$
\begin{aligned}
& \varepsilon^{2} c_{20 ; j}\left(\partial_{s} e_{j}^{(n)}, \partial_{s} e_{j}^{(n)}\right)-\frac{1}{2} \varepsilon\left(e_{j}^{(n)}, e_{j}^{(n)} \partial_{x} p_{j}\right)=\varepsilon^{2}\left[\frac{c_{11 ; j}}{2 h_{1, t}}\left(e_{j}^{(n)}, \partial_{s} e_{j+1}^{(n-1)}-\partial_{s} e_{j-1}^{(n-1)}\right)\right. \\
& \left.\quad+\frac{c_{02 ; j}}{h_{1, t}^{2}}\left(e_{j}^{(n)}, e_{j+1}^{(n-1)}-2 e_{j}^{(n-1)}+e_{j-1}^{(n-1)}\right)+\frac{c_{01 ; j}}{2 h_{1, t}}\left(e_{j}^{(n)}, e_{j+1}^{(n-1)}-e_{j-1}^{(n-1)}\right)\right] .
\end{aligned}
$$

We find a lower bound for the quantity in the left member in the same way as before,

$$
\left|\varepsilon^{2} c_{20 ; j}\left(\partial_{s} e_{j}^{(n)}, \partial_{s} e_{j}^{(n)}\right)-\frac{1}{2} \varepsilon\left(e_{j}^{(n)}, e_{j}^{(n)} \partial_{x} p_{j}\right)\right| \geq \frac{1}{2} \varepsilon^{2}\left(\partial_{s} e_{j}^{(n)}, \partial_{s} e_{j}^{(n)}\right)
$$

The expression inside the brackets in the right member of (4.43) satisfies a uniform estimate of the type

$$
\begin{gathered}
\mid\left[\ldots \| \leq C \varepsilon^{2} a(\varepsilon)\left[h_{1, t}^{-1}\left\|\partial_{s} e^{(n-1)}\right\|_{L^{2}\left(\Omega_{1}\right)}\right.\right. \\
\left.+4 h_{1, t}^{-2}\left\|e^{(n-1)}\right\|_{L^{2}\left(\Omega_{1}\right)}+h_{1, t}^{-1}\left\|e^{(n-1)}\right\|_{L^{2}\left(\Omega_{1}\right)}\right]\left\|\partial_{s} e^{(n)}\right\|_{L^{2}\left(\Omega_{1}\right)} .
\end{gathered}
$$

The desired energy estimate follows from (4.43), (4.44), and (4.45),

$$
\frac{1}{2}\left\|\partial_{s} e^{(n)}\right\|_{L^{2}\left(\Omega_{1}\right)} \leq C a(\varepsilon)\left[h_{1, t}^{-1}\left\|\partial_{s} e^{(n-1)}\right\|_{L^{2}\left(\Omega_{1}\right)}+4 h_{1, t}^{-2}\left\|e^{(n-1)}\right\|_{L^{2}\left(\Omega_{1}\right)}+h_{1, t}^{-1}\left\|e^{(n-1)}\right\|_{L^{2}\left(\Omega_{1}\right)}\right] .
$$

If we compare this inequality with the earlier estimate (4.34), we notice that the upper bound contains a new term involving the $L^{2}$-norm of the $s$-derivative of $e^{(n-1)}$. It is not possible to estimate this term in terms of the norm of $e^{(n-1)}$; cf. (4.35). We therefore take a different approach, estimating the norms of $e^{(n-1)}$ in terms of the norm of $\partial_{s} e^{(n-1)}$,

$$
\frac{1}{2}\left\|\partial_{s} e^{(n)}\right\|_{L^{2}\left(\Omega_{1}\right)} \leq C a(\varepsilon)\left[h_{1, t}^{-1}+\left(4 h_{1, t}^{-2}+h_{1, t}^{-1}\right) a(\varepsilon)\right]\left\|\partial_{s} e^{(n-1)}\right\|_{L^{2}\left(\Omega_{1}\right)}
$$

This result carries over to the discrete case. It shows that the amplification factor is bounded by a constant multiple of $a(\varepsilon)\left[h_{1, t}^{-1}+\left(h_{1, t}^{-2}+h_{1, t}^{-1}\right) a(\varepsilon)\right]$. The latter quantity is of order $\varepsilon^{1 / 2} \log \left(\varepsilon^{-1}\right)$ on $\Omega_{h(\varepsilon)}$. Hence,

$$
\rho_{1}(\varepsilon)=O\left(\varepsilon^{1 / 2} \log \left(\varepsilon^{-1}\right)\right) \text { on } \Omega_{h(\varepsilon)} .
$$

After $n$ steps of the iterative procedure, we have

$$
\left\|\partial_{s} e^{(n)}\right\|_{X\left(\Omega_{1}\right)} \leq a(\varepsilon)^{1 / 2}\left\|\partial_{s} e^{(n)}\right\|_{L^{2}\left(\Omega_{1}\right)} \leq a(\varepsilon)\left(\rho_{1}(\varepsilon)\right)^{n}\left\|\partial_{s} e^{(0)}\right\|_{L^{2}\left(\Omega_{1}\right)},
$$

which shows that the method also converges in the sup norm. The convergence rate is the same and of the order of $\varepsilon^{1 / 2} \log \left(\varepsilon^{-1}\right)$ as $\varepsilon \downarrow 0$. 
The price one pays for the use of a nonorthogonal coordinate system is that the convergence rate is of the order of $\varepsilon^{1 / 2} \log \left(\varepsilon^{-1}\right)$, instead of $\varepsilon \log \left(\varepsilon^{-1}\right)$. On the other hand, with this nonorthogonal coordinate system the solution in the boundary layer is computed on a Cartesian grid that is imbedded in the global Cartesian grid. In the example of the next section, we demonstrate that this approach applies to ordinary transition layers as well. Because the location of a transition layer is usually not known a priori, it is useful to have a Cartesian grid that does not vary in the direction normal to the transition layer. Also, it is easy to compute an approximation in the direction tangential to the transition layer, because the first-order derivative of the solution is of minimal order in this direction. For ordinary transition layers, the derivatives in the direction tangential to the front are of order one, and the mesh width $h_{1, t}$ must be of same order as the mesh width in the regular domain, $h_{1, t} \approx \varepsilon\left(\log \left(\varepsilon^{-1}\right)\right)^{-1}$.

We note that the method used in this section to prove the convergence of the iterative scheme does not work in the case of a singularly perturbed zero-order equation, $-\varepsilon \Delta u+q u=$ $f$, on a general domain $\Omega$. In general, therefore, we cannot conclude that the use of a nonorthogonal grid leads to a convergent algorithm for ordinary layers.

\section{A Singularly Perturbed Turning-Point Problem}

We demonstrate the application of the method to a two-dimensional turning point problem,

$$
-\varepsilon \Delta u+p(x, y) \partial_{x} u=0 \text { on } \Omega, B u=g \text { on } \partial \Omega,
$$

with $\Omega=(-2,2) \times(-1,1)$, where a mixture of Dirichlet and Neumann conditions is imposed on the boundary,

$$
\begin{gathered}
(B u)(-2, y)=u(-2, y)=-1,(B u)(2, y)=u(2, y)=1, \quad-1 \leq y \leq 1 \\
\left.(B u)(x,-1)=\partial_{y} u(x,-1)=0,(B u)(x, 1)=\left(-\partial_{x} u+\partial_{y}\right) u\right)(x, 1)=0, \quad-2 \leq x \leq 2 .
\end{gathered}
$$

The coefficient $p$ changes sign in $\Omega$,

$$
p(x, y)= \begin{cases}-x & \text { if } y \leq 0 \\ -x-y & \text { if } y \geq 0\end{cases}
$$

Here, our interest is not so much in the theoretical analysis as in the numerical results. The analysis of Section 3 needs modification at several points, because the estimates of the propagation factor and the amplification numbers are more complicated. We will not go into these details here. (A variant of the problem (5.1) was studied in [25].)

The solution of (5.1) has a transition layer inside $\Omega$ along the piecewise linear curve $S$,

$$
S=\{(x, y) \in \Omega: x=0 \text { if } y \leq 0, x=-y \text { if } y \geq 0\} .
$$


An asymptotic analysis shows that $u_{\Omega}(\varepsilon)=-1+o(1)$ to the left of the transition layer, and $u_{\Omega}(\varepsilon)=1+o(1)$ to the right of the transition layer as $\varepsilon \downarrow 0$. The transition layer has a complex structure. Away from the origin, it is essentially a one-dimensional transition layer, so only the transverse coordinate needs to be stretched, but near the origin, it is a true corner layer (codimension 2), and both coordinates need to be stretched simultaneously. The corner layer variables are $\xi=\varepsilon^{-1 / 2} x$ and $\eta=\varepsilon^{-1 / 2} y$.

The domain decomposition now involves several different subdomains,

$$
\Omega=\Omega_{0} \cup \Omega_{1} \cup \Omega_{2}
$$

where each subdomain is in turn the union of two disjoint components,

$$
\begin{gathered}
\Omega_{0}=\Omega_{0-} \cup \Omega_{0+}, \quad \Omega_{0 \pm}=\left\{(x, y) \in \Omega: \begin{array}{c}
-2<x<\phi(y)-b \\
\phi(y)+b<x<2
\end{array},-1<y<1\right\} \\
\Omega_{1}=\Omega_{1-} \cup \Omega_{1+}, \quad \Omega_{1 \pm}=\left\{(x, y) \in \Omega:|x-\phi(y)|<a, \begin{array}{l}
y>b \\
y<-b
\end{array}\right\}, \\
\Omega_{2}=\{(x, y) \in \Omega:|x-\phi(y)|<a,-a<y<a\},
\end{gathered}
$$

Here, $0<b<a \ll 1 ; a$ and $b$ satisfy the asymptotic relations

$$
a(\varepsilon) \approx b(\varepsilon) \approx \varepsilon^{1 / 2} \log \left(\varepsilon^{-1}\right) .
$$

We impose a regular grid on $\Omega$, which we refine in the transition layer, and use the same finite-difference approximations for the Laplacian and the partial $x$-derivative as in Section 4 , so the approximation is first-order accurate in $x$, second-order accurate in $y$. Asymptotically, the mesh widths satisfy the relations

$$
h_{0}(\varepsilon) \approx\left(h_{x}(\varepsilon), h_{y}(\varepsilon)\right), h_{1}(\varepsilon) \approx\left(\varepsilon^{1 / 2} h_{x}(\varepsilon), h_{y}(\varepsilon)\right), h_{2}(\varepsilon) \approx\left(\varepsilon^{1 / 2} h_{x}(\varepsilon), \varepsilon^{1 / 2} h_{y}(\varepsilon)\right),
$$

where

$$
h_{x}(\varepsilon) \approx \varepsilon \log \left(\varepsilon^{-1}\right), h_{y}(\varepsilon) \approx \varepsilon^{1 / 2} \log \left(\varepsilon^{-1}\right) .
$$

The truncation error is linear in $\varepsilon$, apart from a logarithmic factor.

In the Schwarz procedure, we alternate the computation on the regular domain, $\Omega_{0}$, with the computation in the layer, $\Omega_{1} \cup \Omega_{2}$. In the layer, in turn, we alternate the computation away from the origin, in $\Omega_{1}$, with the computation in the corner layer near the origin, $\Omega_{2}$.

On the regular domain, $\Omega_{0}$, we take a cut-off function $\chi$, which is one in the domain of overlap with the layer and which decays exponentially to zero in the regular domain away from the layer. On $\Omega_{0}$, convection dominates. The iterative procedure could be based on the same decomposition as in Section $4.1,-\varepsilon \chi(\varepsilon) \Delta u+p(x, y) \partial_{x} u=\varepsilon(1-\chi(\varepsilon)) \Delta u$, or on the even simpler decomposition $p(x, y) \partial_{x} u=\varepsilon \Delta u$. However, to benefit from the convergence 
of the Schwarz procedure, it is preferable to retain part of the perturbation, at least in the domain of overlap. We have therefore implemented a simplified version,

$$
-\varepsilon \chi(\varepsilon) \partial_{x}^{2} u+p(x, y) \partial_{x} u=\varepsilon(1-\chi(\varepsilon)) \partial_{x}^{2} u+\varepsilon \partial_{y}^{2} u
$$

where only the second-order derivative with respect to $y$ is treated explicitly. This procedure leads to a nicely parallel algorithm. Still better would be to use a nonorthogonal decomposition of the Laplacian in $\Omega_{0} \cap\{y \geq 0\}$. In either case, we can apply Dirichlet boundary conditions on the interfaces in the transition layer.

The transition layer is essentially a one-dimensional phenomenon. On $\Omega_{1-}$, the part of the transition layer in the lower half plane, the $x$ direction is normal to the front; $x$ needs to be stretched, but $y$ does not. The iterative scheme is based on the splitting

$$
-\varepsilon \partial_{x}^{2} u+p(x, y) \partial_{x} u=\varepsilon \partial_{y}^{2} u .
$$

Although a poor initial guess for the Schwarz procedure may lead to a second-order derivative with respect to $y$ that is large in the overlap region with $\Omega_{2-}$, it is our experience that the solution straigthens out rapidly. On $\Omega_{1+}$, the part of the transition layer in the upper half plane, the normal direction to the front is $(1,1)^{t}$. The first-order term of the expansion of the exact solution is insensitive to the direction of the stretched variable as long as this direction is transverse to the front. The other space direction used in the change of variables must be tangential to the front; otherwise, the second-order term of the formal expansion is of the same order as the first-order term, and the expansion is not valid. We therefore make a change of variables, $s=x+y, t=y$. In the $(s, t)$-coordinate system, the Laplacian is represented by $2 \partial_{s}^{2} u+2 \partial_{s} \partial_{t} u+\partial_{t}^{2} u$. We then base the iterative scheme on the splitting

$$
-2 \varepsilon \partial_{s}^{2} u+p(x, y) \partial_{s} u=\varepsilon\left(2 \partial_{s} \partial_{t} u+\partial_{t}^{2} u\right)
$$

On $S$, we have $s=0$, so it is easy to obtain a finite-difference approximation of the cross derivative and the second-order derivative with respect to $t$. In fact, we chose $S$ as in (5.5) precisely to obtain a two-dimensional turning point problem with a very elementary numerical implementation. In principle, however, there is no restriction on the curve $S$ as long as the angle between the tangent to $S$ and the coordinate direction that is stretched is at least $\frac{1}{2} \pi$.

Finally, in the corner layer, $\Omega_{2}$, the derivatives with respect to both $x$ and $y$ are treated implicitly. The approximation scheme leads to a sparse matrix that is irreducible and diagonally dominant. Since the mesh on $\Omega_{2}$ is a local refinement of the global mesh by a factor $\varepsilon^{1 / 2}$, and we need at least four points of the global mesh across $\Omega_{2}$, the number of mesh points on $\Omega_{2}$ is at least $16 \varepsilon^{-1}$. Thus, even though the size of the corner layer is small, the fact that we treat both $x$ and $y$ derivatives implicitly on $\Omega_{2}$ puts an effective lower bound on the values of $\varepsilon$. 


\section{$5.1 \quad$ Numerical Results}

We have implemented the method outlined in the present article and solved the singular perturbation problem (5.1) with various parameter values. Here, we comment on the solution obtained with $\varepsilon=0.005, N_{x}=N_{y}=10$, a mesh refinement factor of 10 in the layer, and a minimum overlap between the various subdomains. The mesh widths are $h_{0, x}=h_{0, y}=0.2$ on the regular domain (coarse grid), $h_{1, x}=0.02, h_{1, y}=0.2$ in the transition layer, away from the origin; and $h_{2, x}=h_{2, y}=0.02$ in the corner layer. The cut-off function $\chi$ involved in the solution on $\Omega_{0}$ is one in the overlap with the layer, extends two mesh cells into the regular domain, and vanishes beyond. We used seven Schwarz iterations to alternate between $\Omega_{0}$ and the layer ( $m=7$ in Theorem 2), and two Schwarz iterations within the layer to alternate between $\Omega_{1}$ and $\Omega_{2}(m=2$ in Theorem 2$)$. On $\Omega_{0}$ and $\Omega_{1}$, we made three iterations on each subdomain, $\left(n_{0}=n_{1}=3\right.$ in Theorem 2)); on $\Omega_{2}$, we solved the linear system directly. Thus, the total number of steps was 14 on $\Omega_{0}, 42$ on $\Omega_{1}$, and 21 on $\Omega_{2}$.

Figure 1 gives the solution computed on the coarse grid. The front is sharp, but the resolution is clearly inadequate in the layer. Figure 2 complements Figure 1 and shows the solution on the fine grid in the transition layer. Figure 3 gives the solution in the various subdomains. Figure 4 illustrates the global convergence; the ratio $\left\|u^{(m+1)}-u^{(m)}\right\|_{X(\Omega)} / \| u^{(m)}-$ $u^{(m-1)} \|_{X(\Omega)}$ in successive steps $(m)$ of the Schwarz alternating procedure is approximately 0.09 . The same calculation with $\varepsilon=0.01$, when the refinement factor is exactly 10 , resulted in slower convergence. The observed convergence rate was 0.15 , which is consistent with the statement that the convergence rate is of the order of $\left(\log \left(\varepsilon^{-1}\right)\right)$. Figure 5 shows the convergence record on the various subdomains.

Convergence is very fast in all cases. Also, the smaller the ratios $\varepsilon / h_{0}$ and $\varepsilon / h_{2}$, the faster the convergence. Of course, these ratios must not be too small, because otherwise the solution in the corner layer becomes unreasonably expensive. The choice $h_{0}=0.2$ and $h_{2}=0.02$ for $\varepsilon=0.005$ is a good compromise.

The iterative procedure in $\Omega_{1}$ is not doing well at the beginning of the iterative process. Our initial condition for the Schwarz procedure was a numerical solution of the problem, obtained with a direct method and $\varepsilon=0.1$. In the layer, this solution is quite far from the solution with $\varepsilon=0.005$. Consequently, the solution varies rapidly in the overlap region $\Omega_{1} \cap \Omega_{2}$ at the beginning of the iteration process. The second-order derivative of the solution in the direction tangential to the front is not small, and the splitting of the operator is not very efficient.

The method has a high degree of parallelism at the domain-decomposition level and at the level of the iterative procedures on each subdomain. At the domain-decomposition level, however, the method does not scale. One may wish to use classical domain decomposition in the regular domain for convection-dominant problems and in the corner layer for elliptic problems. We have not explored these options. In the transition layer, one could use a line 
of processors to solve each ordinary differential equation in parallel. In the regular domain, we have the classical method of lines with the same type of one-dimensional structure for parallelism. Nevertheless, it is difficult to implement the proposed method efficiently on a parallel architecture. In contrast to the approach first proposed in [25], we no longer use the same scheme on each subdomain. Therefore, simply keeping the degrees of freedom the same on each subdomain will not guarantee proper load balancing. The use of an asynchronous method may seem appropriate at first sight, but, in fact, a totally asynchronous version hides the difficulty. The solution will certainly converge faster on some subdomains, so every compute node can be kept busy, but the efficiency will not be balanced evenly. With a fixed geometry of the subdomains and a fixed number of iterations on each subdomain, as in the turning-point example, it is relatively easy to compute a priori the computational cost of each procedure and balance the tasks on a network of processors. Whether one can implement a heterogeneous domain-decomposition method efficiently with a dynamic load-balancing procedure on a massively parallel architecture is still an open question.

\section{Appendix A Some Concepts of Asymptotic Analysis}

In this appendix we summarize some relevant results of asymptotic analysis and singular perturbation theory. For details, we refer the reader to our notes $[31,32]$

Order relations for scalar-valued functions. Two positive-valued functions $f$ and $g$, defined and continuous on some interval $\left(0, \varepsilon_{0}\right)$ with $\varepsilon_{0}>0$, satisfy the asymptotic order relation $f=O(g)$ as $\varepsilon \downarrow 0$ if there exists an $\varepsilon_{0}>0$ and a positive constant $C$, which may depend on $\varepsilon_{0}$ but not on $\varepsilon$, such that $f(\varepsilon)<C g(\varepsilon)$ for all $\varepsilon \in\left(0, \varepsilon_{0}\right) ; f=o(g)$ as $\varepsilon \downarrow 0$ if, for every positive constant $c$, there exists an $\varepsilon_{0}>0$, which may depend on $c$, such that $f(\varepsilon)<c g(\varepsilon)$ for all $\varepsilon \in\left(0, \varepsilon_{0}\right) ; f=O^{\sharp}(g)$ if $f=O(g)$ and $f \neq o(g) ; f \approx g$ if $f=O(g)$ and $g=O(f) ; f \sim g$ if $f-g=o(1)$.

Order relations for vector-valued and variable functions. The asymptotic order relations carry over to vector-valued functions. Suppose $X$ and $Y$ are normed vector spaces of functions defined on the domains $\Omega_{X}$ and $\Omega_{Y}$, respectively. Let $u:\left(0, \varepsilon_{0}\right) \rightarrow X$ and $v:\left(0, \varepsilon_{0}\right) \rightarrow Y$ be such that the mappings $f: \varepsilon \mapsto\|u(\varepsilon)\|_{X}$ and $g: \varepsilon \mapsto\|v(\varepsilon)\|_{Y}$ are continuous on some interval $\left(0, \varepsilon_{0}\right)$. Then $u=O(v)$ as $\varepsilon \downarrow 0$ if $f=O(g)$ as $\varepsilon \downarrow 0$, et cetera. Variable functions are covered through the identifications $u(\varepsilon)(x)=u(\varepsilon, x)$ for all $x \in \Omega_{X}$, $v(\varepsilon)(y)=v(\varepsilon, y)$ for all $y \in \Omega_{Y}$.

Asymptotic approximations. Asymptotic approximations are defined with respect to a "gauge" - that is, an asymptotically ordered set of order functions. An order function is a 
positive-valued monotone function on $\left(0, \varepsilon_{0}\right)$. A gauge is a set $\mathcal{E}=\left\{\delta_{n}(\varepsilon): n=0,1, \ldots\right\}$ of order functions $\delta_{n}$ on $\left(0, \varepsilon_{0}\right)$, which satisfy the order relation $\delta_{n+1}=o\left(\delta_{n}\right)$ for $n=0,1, \ldots$ as $\varepsilon \downarrow 0$. In most applications, one has $\delta_{n}=\delta^{n}$ for $n=0,1, \ldots$, where $\delta$ is a fixed-order function; for example, $\delta(\epsilon)=\varepsilon^{\nu}$ with $\nu>0$, or $\delta(\varepsilon)=\left(\log \left(\varepsilon^{-1}\right)\right)^{-1}$.

Given two mappings $u, u_{n}:\left(0, \varepsilon_{0}\right) \rightarrow X$, we say that $u_{n}$ is an asymptotic approximation of order $\delta_{n}$ if $u-u_{n}=o\left(\delta_{n}\right)$ as $\varepsilon \downarrow 0$.

Regular asymptotic expansions. A regular asymptotic expansion is an asymptotic approximation of the form $u_{n}(\varepsilon)=\sum_{i} \delta_{i}(\varepsilon) v_{i}$, where $v_{i} \in X, v_{i}$ independent of $\varepsilon$. The coefficients $v_{i}$ in a regular asymptotic expansion are defined with respect to the specific gauge; they are unique and can be determined successively by taking limits in $X$.

If a function $u$ admits a regular asymptotic expansion to any order, we say that the sequence $\left\{u_{n}: n=0,1, \ldots\right\}$ converges asymptotically to $u$ as $\varepsilon \downarrow 0$. Notice that asymptotic convergence is much weaker than ordinary convergence, which concerns the behavior of the sequence as $n \rightarrow \infty$ at a fixed value of $\varepsilon$. An asymptotically convergent sequence need not converge in the ordinary sense.

Singular perturbation problems. Of particular interest for applications are asymptotic expansions in spaces of continuous functions, where convergence means "uniform convergence." The coefficients $v_{i}$ in a regular asymptotic expansion are then obtained as uniform limits of continuous functions and are therefore themselves continuous. In singular perturbation problems, the limiting procedure breaks down, because of a lack of uniformity, and singular (boundary and transition) layers develop as $\varepsilon \downarrow 0$. The limiting procedure works on compact subsets of $\Omega$ ("regular subdomains"), but not on $\bar{\Omega}$, and a separate asymptotic analysis is needed in the singular layers.

Singular layer analysis. In many instances, it is possible to regularize the singular boundary value problem locally in a singular layer. The regularization requires the existence of a transformation $\tau(\varepsilon): x \mapsto \xi$, defined for all $\varepsilon \in\left(0, \varepsilon_{0}\right)$, which is monotone, one-to-one, invertible, and which stretches the transverse coordinate across the singular layer "in just the right way."

Suppose the singular layer is a boundary layer and is adjacent to a smooth manifold $\Gamma$ (an open subset of the boundary $\partial \Omega$ ). The transformation $\tau(\varepsilon)$ maps $\Omega$ one-to-one onto $\tau(\varepsilon)(\Omega)$ and establishes a continuous mapping between $X(\Omega)$ and $X(\tau(\varepsilon)(\Omega))$. In particular, it sends the solution $u_{\Omega}(\varepsilon)$ of (1.1) into a function $U(\varepsilon)$,

$$
\dot{U}(\varepsilon)(\xi)=U(\varepsilon, \xi)=u_{\Omega}(\varepsilon, x), \xi=\tau(\varepsilon) x, x \in \Omega .
$$


The domains $\tau(\varepsilon)(\Omega)$ are nested: $\tau\left(\varepsilon_{1}\right)(\Omega) \subset \tau\left(\varepsilon_{2}\right)(\Omega)$ if $\varepsilon_{1}>\varepsilon_{2}$. The union,

$$
\tau(\Omega)=U_{\varepsilon \in\left(0, \varepsilon_{0}\right)} \tau(\varepsilon)(\Omega),
$$

is a subset of $R^{d}$, which is unbounded in the directions transverse to the singular part of the boundary. The set $X(\tau(\Omega))$ of all continuous functions on $\tau(\Omega)$, endowed with the inductive limit topology,

$$
X(\tau(\Omega))=\lim \operatorname{ind}_{\varepsilon \downarrow 0} X(\tau(\varepsilon)(\Omega)),
$$

is a complete normed vector space [33, Section 50]. This space is the proper framework for the asymptotic analysis of the function $U(\varepsilon)$. Convergence in $X(\tau(\Omega))$ is uniform convergence on compact subsets of $\tau(\Omega)$.

The family of stretching transformations $\tau=\left\{\tau(\varepsilon): \varepsilon \in\left(0, \varepsilon_{0}\right)\right\}$ is a regularizing transformation for (1.1) in the boundary layer if, first of all, there exists a gauge with respect to which $U(\varepsilon)$ admits a regular asymptotic expansion on any ( $\varepsilon$-independent) compact subset of $\tau(\Omega)$, and if, furthermore, the domain of validity of this regular asymptotic expansion extends to any compact subset of $\tau(\Omega \cup \Gamma)$. The boundary layer is the pre-image of any such compact subset, and the stretched coordinates $\xi=\tau(\varepsilon) x$ defined by the regularizing transformation are the "boundary layer variables." The regular asymptotic expansion of $U(\varepsilon)$ yields an asymptotic expansion (though not a regular asymptotic expansion) of $u_{\Omega}(\varepsilon)$ in the boundary layer. This expansion must then be matched to the regular asymptotic expansion in the regular subdomain.

\title{
DISCLAIMER
}

\begin{abstract}
This report was prepared as an account of work sponsored by an agency of the United States Government. Neither the United States Government nor any agency thereof, nor any of their emplayees, makes any warranty, express or implied, or assumes any legal liability or responsibility for the accuracy, completeness, or usefulness of any information, apparatus, product, or process disclosed, or represents that its use would not infringe privately owned rights. Reference herein to any specific commercial product, process, or service by trade name, trademark, manufacturer, or otherwise does not necessarily constitute or imply its endorsement, recommendation, or favoring by the United States Government or any agency thereof. The views and opinions of authors expressed herein do not necessarily state or reflect those of the United States Government or any agency thereof.
\end{abstract}




\section{References}

[1] W. Eckhaus, Matched Asymptotic Expansions and Singular Perturbations, Mathematics Studies \#6, North-Holland, Amsterdam, 1973.

[2] W. Eckhaus, Asymptotic Analysis of Singular Perturbations, Studies in Mathematics and Its Applications \#9, North-Holland, Amsterdam, 1979.

[3] W. Eckhaus \& E. M. de Jager, eds., Theory and Applications of Singular Perturbations, Lect. Notes in Math. \#942, Springer-Verlag, New York, 1982.

[4] R. E. O'Malley, Jr., ed., Asymptotic Methods and Singular Perturbations, SIAM-AMS Proc. Symposia in Applied Mathematics \#10, American Mathematical Society, Providence, R.I., 1976.

[5] R. E. O'Malley, Jr., Introduction to Singular Perturbations, Academic Press, New York, N.Y., 1974.

[6] R. E. O'Malley, Jr., Singular Perturbation Methods for Ordinary Differential Equations, Applied Mathematical Sciences \#89, Springer-Verlag, New York, 1991.

[7] D. R. Smith, Singular Perturbation Theory, Cambridge University Press, Cambridge, 1985.

[8] R. C. Y. Chin, G. W. Hedstrom, J. R. Graw, \& F. A. Howes, "Parallel computation of multiple scale problems," in New Computing Environments: Parallel, Vector and Systolic, A. Wouk, ed., Society for Industrial and Applied Mathematics, Philadelphia, 1986, 136-153.

[9] A. Bourgeat \& M. Garbey, "Computation of viscous or nonviscous conservation laws by domain decomposition based on asymptotic analysis," Num. Methods for PDEs 8(1992), $127-142$.

[10] F. Brezzi, C. Canuto, \& A. Russo, "A self-adaptive formulation for the Euler/NavierStokes coupling," Comp. Meth. in Appl. Mech. and Eng. 73 (1989), 317-330.

[11] G. W. Hedstrom \& F. A. Howes, "A domain decomposition method for a convectiondiffusion equation with turning point," in Domain Decomposition Methods, T. Chan, R. Glowinski, J. Periaux, \& O. Widlund, eds., Society for Industrial and Applied Mathematics, Philadelphia, 1989, 38-46. 
[12] M. K. Kadalbajoo \& Y.N. Reddy, "Asymptotic and numerical analysis of singular perturbation problems: A survey," Appl. Math. and Comp.30(1989), 223-259.

[13] Yu. A. Kuznetsov, "Overlapping domain decomposition methods for finite element methods with singular perturbed elliptic operators," in Proc. 4th Int. Symp. on Domain Decomposition Methods for Partial Differential Equations, R. Glowinsky, Yu. Kuznetsov, G. Meurant, J. Periaux, \& O. Widlund, eds., Society for Industrial and Applied Mathematics, Philadelphia, 1991, 223-241.

[14] A. Quarteroni, F. Pasquarelli \& A. Vally, "Heterogeneous domain decomposition: Principles, Algorithms, Applications," in Proc. 5th Int. Symp. on Domain Decomposition Methods for Partial Differential Equations, T. F. Chan, D. E. Keyes, G. Meurant, J. S. Scroggs, \& R. G. Voigt, eds., Society for Industrial and Applied Mathematics, Philadelphia, 1992, 129-150.

[15] Wei Pai Tang, "Numerical solution of a turning point problem," in Proc. 5th Int. Symp. on Domain Decomposition Methods for Partial Differential Equations, T. F. Chan, D. E. Keyes, G. Meurant, J. S. Scroggs, \& R. G. Voigt, eds., Society for Industrial and Applied Mathematics, Philadelphia, 1992, 330-338.

[16] H. G. Kaper \& M. Garbey, eds., Asymptotic Analysis and the Numerical Solution of Partial Differential Equations, Lecture Notes in Applied Mathematics \#130, Marcel Dekker, New York, 1991.

[17] H. G. Kaper \& M. Garbey, eds., Asymptotic and Numerical Methods for Partial Differential Equations with Critical Parameters, NATO ASI Series C: Mathematical and Physical Sciences \#384, Kluwer Academic Publishers, Dordrecht (Neth.), 1993.

[18] R. Glowinsky, G. H. Golub, G. A. Meurant, \& J. Periaux, eds., Proc. 1st Int. Symp. on Domain Decomposition Methods for Partial Differential Equations, Society for Industrial and Applied Mathematics, Philadelphia, 1988.

[19] T. F. Chan, R. Glowinsky, J. Periaux, \& O. Widlund, eds., Proc. 2nd Int. Symp. on Domain Decomposition Methods for Partial Differential Equations, Society for Industrial and Applied Mathematics, Philadelphia, 1989.

[20] T. F. Chan, R. Glowinsky, J. Periaux \& O. Widlund, eds., Proc. 3rd Int. Symp. on Domain Decomposition Methods for Partial Differential Equations, Society for Industrial and Applied Mathematics, Philadelphia, 1990.

[21] R. Glowinsky, Yu. Kuznetsov, G. Meurant, J. Periaux, \& O. Widlund, eds., Proc. 4th Int. Symp. on Domain Decomposition Methods for Partial Differential Equations, Society for Industrial and Applied Mathematics, Philadelphia, 1991. 
[22] T. F. Chan, D. E. Keyes, G. Meurant, J. S. Scroggs, \& R. G. Voigt, eds., Proc. 5th Int. Symp. on Domain Decomposition Methods for Partial Differential Equations, Society for Industrial and Applied Mathematics, Philadelphia, 1992.

[23] A. Quarteroni, J. Periaux, Yu. A. Kuznetsov \& O. B. Widlund, eds., Domain Decomposition Methods in Science and Engineering: The Sixth Int. Conf. on Domain Decomposition, June 1992, Contemporary Mathematics \#157, American Mathematical Society, Providence, R.I., 1994.

[24] Zhiqiang Cai \& S. McCormick, "Computational complexity of the Schwarz alternating procedure," Int. J. High-Speed Computing 1(1989), 1-28.

[25] M. Garbey, "A Schwarz alternating procedure for singular perturbation problems," SIAM J. Sci. Comp., (to appear).

[26] F. Desprez \& M. Garbey, "Numerical solution of a combustion problem on a Paragon machine," Parallel Computing 21 (1995), 495-508.

[27] M. Garbey, "Domain decomposition to solve transition layers and asymptotics," SIAM J. Sci. Comp. 15 (1994), 866-891.

[28] A. Bayliss, M. Garbey, \& B. J. M. Matkowsky, "Adaptive pseudo-spectral domain decomposition and the approximation of multiple layers," J. Comp. Phys., (to appear).

[29] Y. Achdou \& O. Pironneau, "The $\chi$ method for the Navier Stokes equations," C. $R$. Académie des Sciences 1(1991), 168.

[30] R. Courant \& D. Hilbert, Methods of Mathematical Physics, John Wiley \& Sons, New York, 1962.

[31] H. G. Kaper \& M. Garbey, Asymptotic Analysis. Working Notes \#1. Basic Concepts and Definitions, ANL/MCS-TM-179, Mathematics and Computer Science Division, Argonne National Laboratory, 1993.

[32] H. G. Kaper \& M. Garbey, Asymptotic Analysis. Working Notes \#3. Boundary Layers, ANL/MCS-TM-181, Mathematics and Computer Science Division, Argonne National Laboratory, 1993.

[33] F. Trèves, Topological Vector Spaces, Distributions and Kernels, Academic Press, New York, 1967. 
Figure captions.

- Figure 1. Solution of (5.1) on coarse grid

- Figure 2. Solution of (5.1) and computational grid in the transition layer

- Figure 3. Solution of (5.1) on the subdomains (clockwise from top left): regular subdomain, $\Omega_{0}$; lower transition layer, $\Omega_{1-}$; corner layer, $\Omega_{2}$; upper transition layer, $\Omega_{1+}$

- Figure 4. Global convergence of the Schwarz alternating procedure; $e(m)=\| u^{(m)}-$ $u^{(m-1)} \|_{X(\Omega)}$.

- Figure 5. Convergence of the iterative procedure on the subdomains (clockwise from top left): corner layer, $\Omega_{2}$; lower transition layer, $\Omega_{1-} ;$ regular subdomain, $\Omega_{0} ;$ upper transition layer, $\Omega_{1+} ; e(n)=\left\|u^{(n)}-u^{(n-1)}\right\|_{X\left(\Omega_{i}\right)}$. 


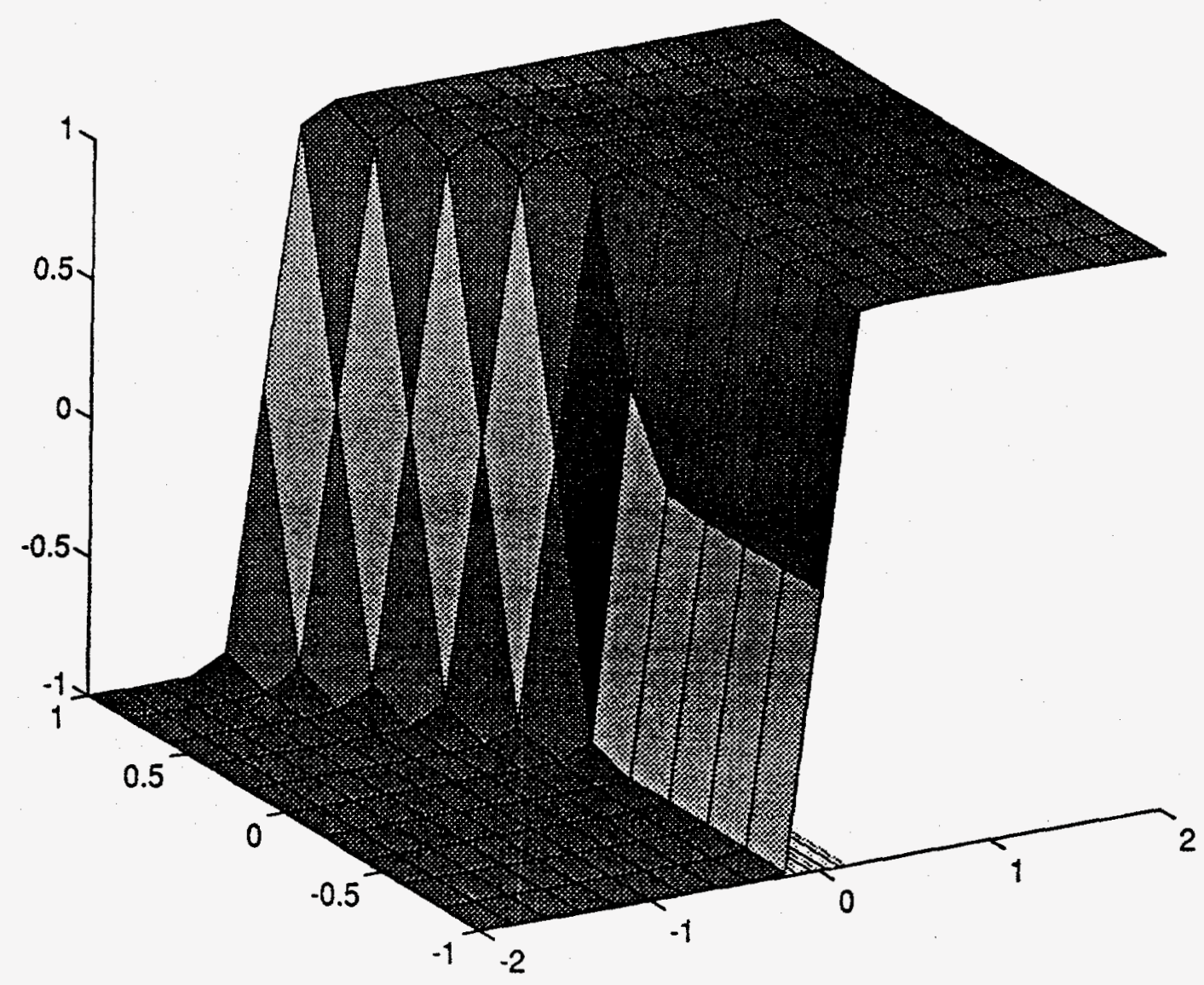

FIGURE 1 


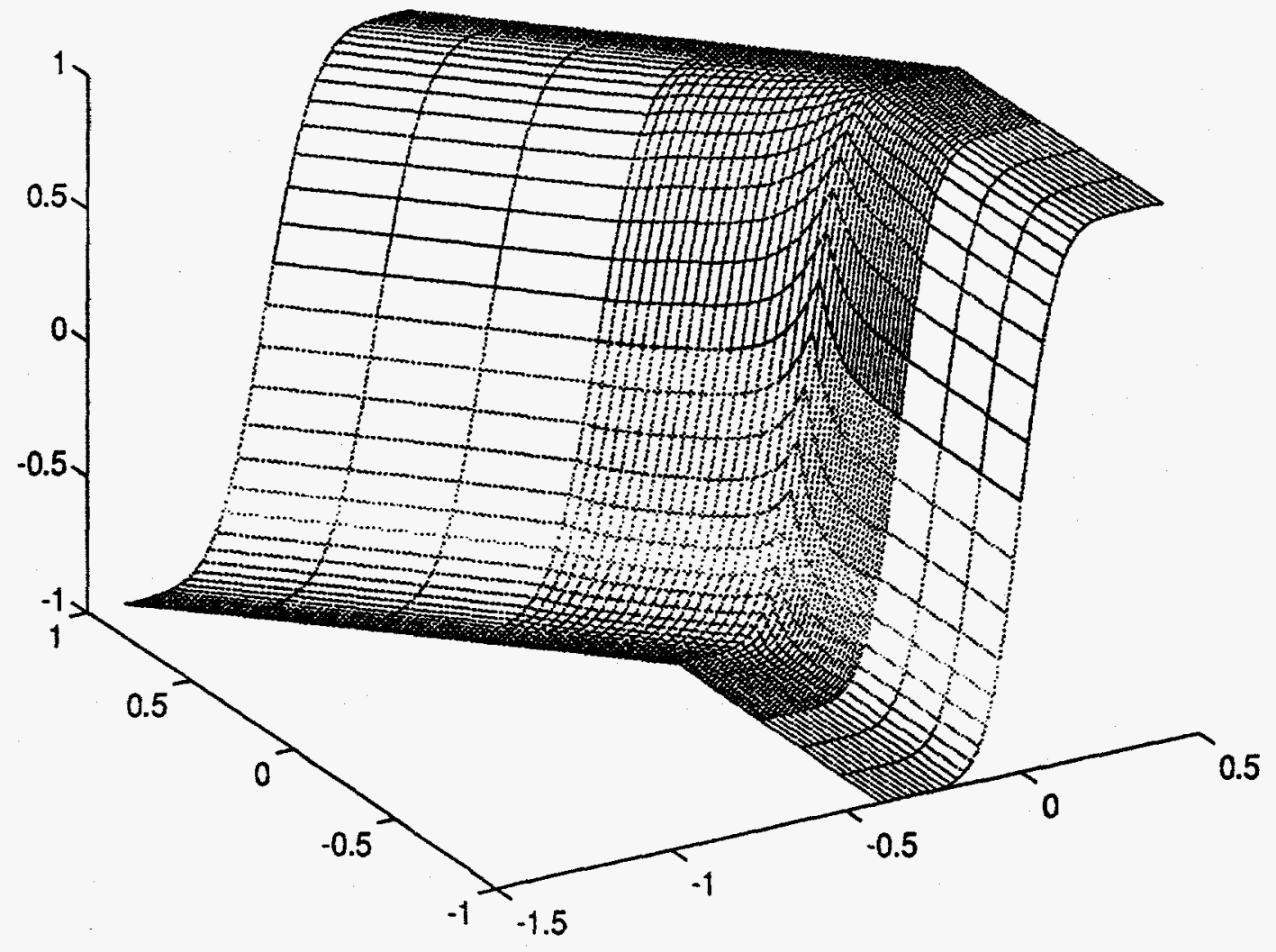

FIGURE 2 

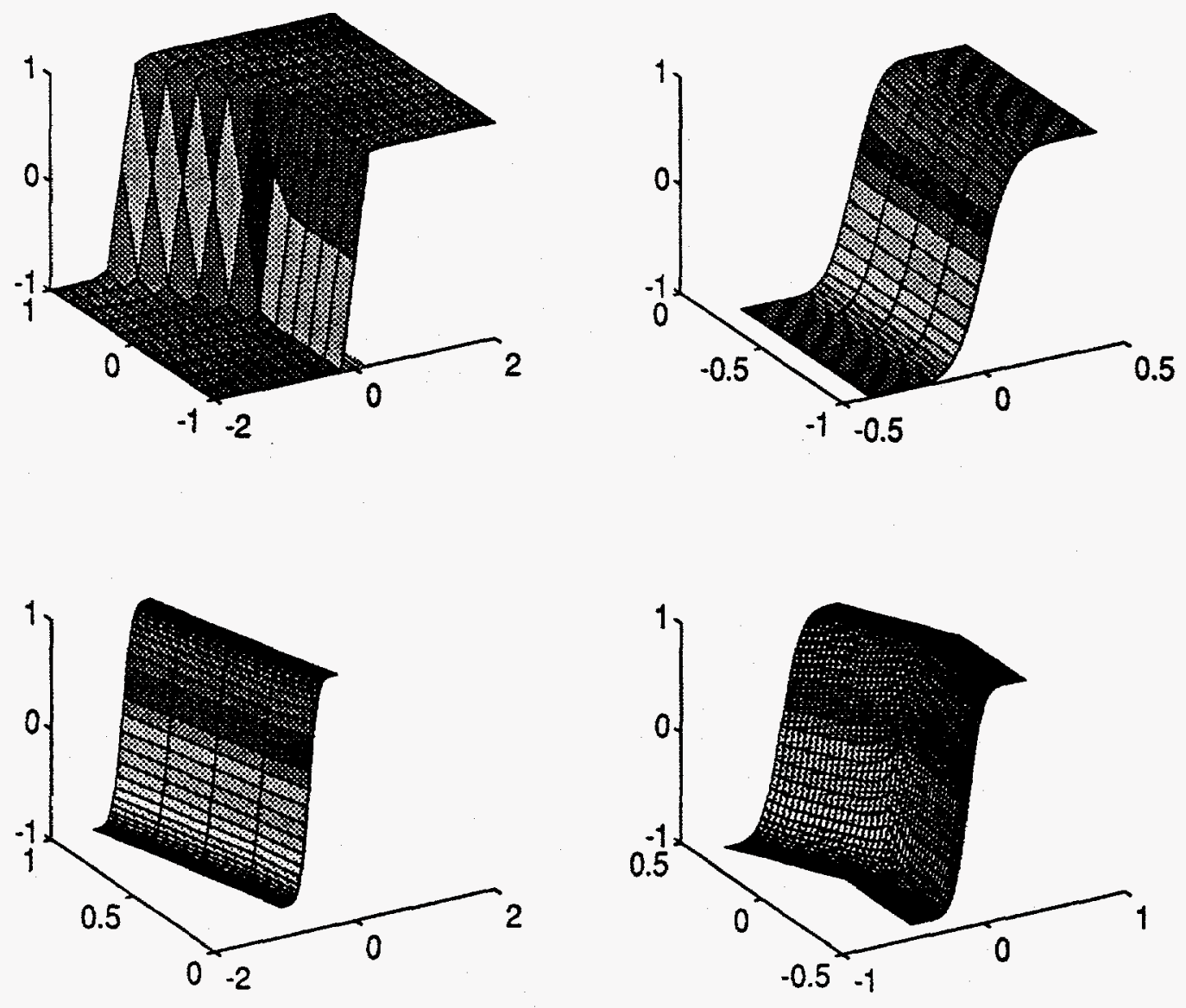

FIGURE 3 


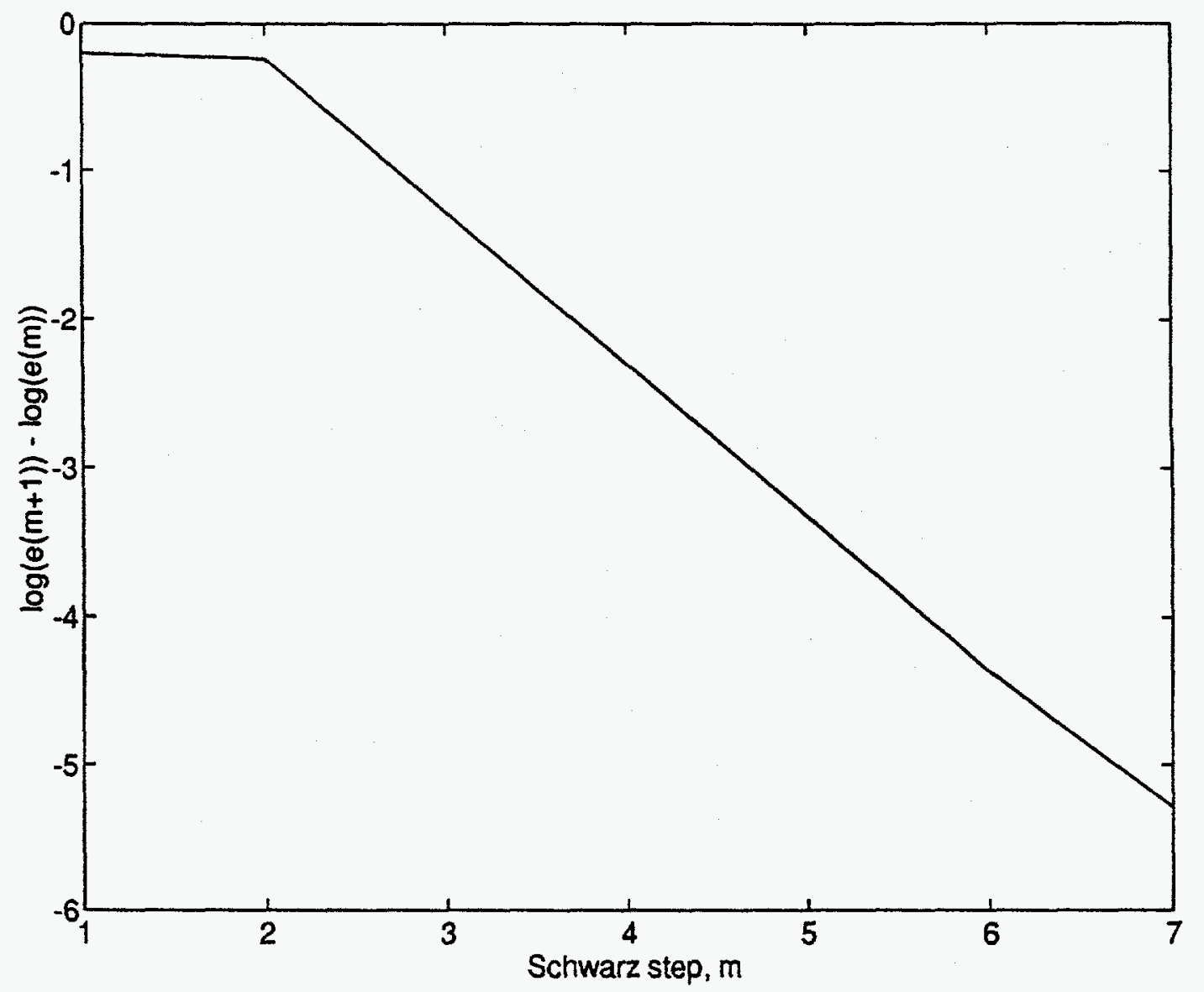

FIGURE 4 

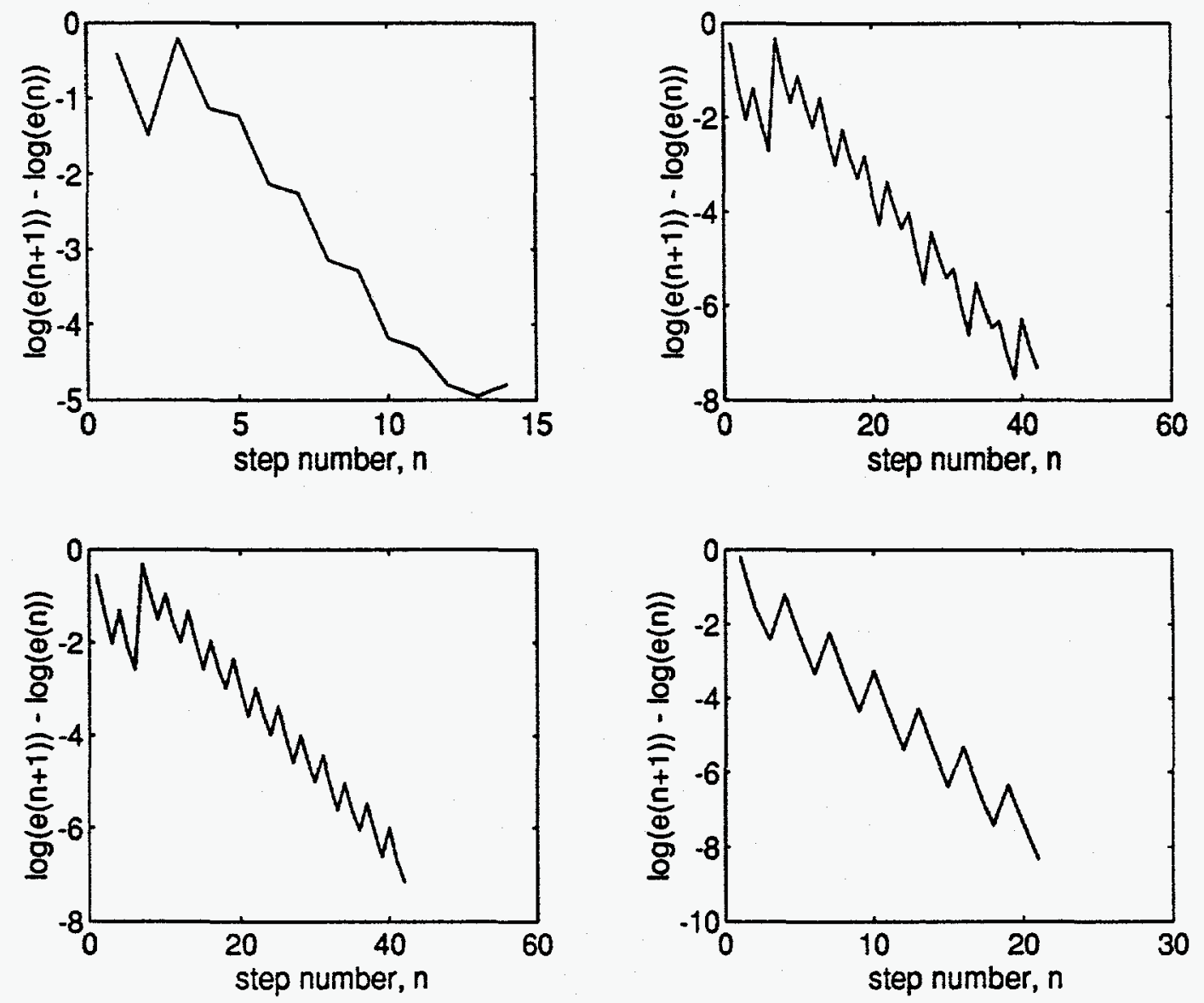

FIGURE 5 


\section{DISCLAIMIER}

Portions of this document may be illegible in electronic image products. Images are produced from the best available original document. 